\title{
Stochastische Unternehmensbewertung mittels Monte-Carlo-Simulation am Beispiel der Verlagsbranche
}

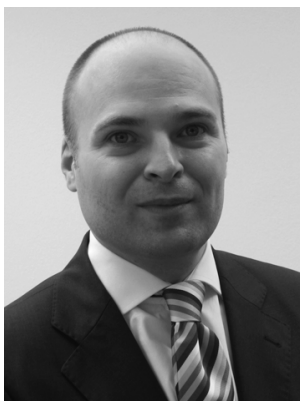

Heinrich H. Förster

Monte-Carlo-Simulation, Risikoanalyse, Werttreiber, Unternehmensbewertung, Discounted Cash Flow (DCF) Verfahren, gewichtete durchschnittliche Kapitalkosten (WACC)

Monte-Carlo simulation, risk analysis, value driver, valuation, discounted cash flow (DCF), weighted average cost of capital (WACC)

Der vorliegende Beitrag verfolgt das Ziel, die Nutzung stochastischer Risikoanalysen in der Unternehmensbewertung aufzuzeigen. Anhand des Beispiels eines fiktiven deutschen Unternehmens aus der Verlagsbranche wird dargestellt, wie unter Rückgriff auf historische Daten Verteilungsannahmen für relevante Werttreiber nach dem Shareholder-Value-Konzept von Rappaport gebildet werden können. Unter Verbindung praktischer und theoretischer Anforderungen wird gezeigt, wie sich die Monte-Carlo-Simulation als Instrument zur Prognose bewertungsrelevanter finanzieller Überschüsse nutzen lässt, die einer Unternehmensbewertung nach der Risikozuschlagsmethode zugeführt werden.

The paper demonstrates the use of stochastic risk analysis for company valuation. Based on an example of a fictitious German company in the newspaper and publishing industry the author illustrates how historical data samples can be used to draw inferences on the statistical distribution of relevant value drivers as defined using Rappaport's shareholder value framework. Taking practical and theoretical requirements into consideration, the author shows how Monte-Carlo-Simulation can be used as an instrument for estimating relevant cash flows, which have to be discounted using risk-adjusted discount rates for company valuation.

\section{Einleitung}

Die Erfassung und Berücksichtigung von Risiken bildet nach wie vor einen zentralen Problemkreis für Theorie und Praxis der Unternehmensbewertung. Seit langem werden im deutschsprachigen Raum die Risikozuschlags-, Sicherheitsäquivalenz- und Risikoprofilmethode diskutiert (vgl. statt vieler Schultze 2003, 259 ff.). Dieser Beitrag verfolgt das Ziel, die Nutzung stochastischer Instrumente wie die Monte-Carlo-Simulation für die Praxis der Unternehmensbewertung aufzuzeigen. Zwar ist die Monte-Carlo-Simulation in der theoretischen Auseinandersetzung zur Unternehmensbewertung ausgiebig behandelt. Die Neuerung des hier vorgestellten Ansatzes besteht in der praktischen Anwendung. Es wird gezeigt, wie unter Nutzung historischer Daten Verteilungsannahmen für relevante Werttreiber gebildet werden können, um diese mittels Monte-Carlo-Simulation zu prognostizieren. 
Das bedeutet, dass die Cash Flows nicht direkt stochastisch bestimmt werden. In der Praxis fehlt es regelmässig an Bezugspunkten für die Ableitung von Verteilungen (und Korrelationen). Auch wird zum Zwecke der Risikoanalyse kein stochastischer Kapitalwert (Bamberg et al. 2006, insbesondere 294 f.) ermittelt und die Monte-Carlo-Simulation nicht als praktische Alternative (Buch/Dorfleitner 2007, 151 f.) zur Bestimmung des Sicherheitsäquivalents eingesetzt. ${ }^{1}$ Die Bewertung erfolgt nach der in der Praxis nach wie vor überwiegend angewendeten Risikozuschlagsmethode (vgl. zur Anwendung dieser Methode Brealey et al. 2008, 250-255 und 529 ff.; Coenenberg 1992, 113 ff.).

Durch die Simulation der Wertreiber wird es für den Bewerter möglich, Einblicke in das Risikoprofil der bewertungsrelevanten finanziellen Überschüsse zu erlangen. Durch die Anbindung an Werttreiber ist ein konkreter Bezug zur Unternehmensplanung hergestellt. Darüber hinaus ist eine Basis zur Erstellung von Vergleichen gegeben. Beide unternehmerischen Aufgaben sind von grosser praktischer Relevanz. Die direkte Verknüpfung mit der Bewertung macht es für Bewerter und Entscheider möglich, die verschiedenen Aufgabenstellungen innerhalb eines konsistenten Rahmens zu bewältigen.

Die Ausführungen dieses Beitrags stützen sich auf ein Fallbeispiel für einen fiktiven nicht-börsennotierten Zeitungs- und Zeitschriftenverlag. Da sich die gesamte Printmedienbranche aufgrund der zunehmenden Verbreitung des Internets im strukturellen Wandel befindet und somit durch hohe Unsicherheit gekennzeichnet ist, lassen sich Potentiale und Grenzen simulationsgestützter Risikoanalysen besonders anschaulich in diesem Kontext darstellen. Zur Bildung der erforderlichen Verteilungsannahmen für die zu identifizierenden Werttreiber wird - vergleichbar mit der Durchführung einer Multiplikatoranalyse und der Bestimmung von Kapitalkosten für nicht-börsennotierte Unternehmen - auf historische Finanzdaten einer Vergleichsgruppe börsennotierter Zeitungs- und Zeitschriftenverlage zurückgegriffen.

Der Beitrag gliedert sich in fünf Abschnitte. Im folgenden Abschnitt 2 wird gezeigt, wie die Analyse, Planung und Prognose von Werttreibern am Beispiel von Zeitungs- und Zeitschriftenverlagen (nachfolgend Verlage, Verlagsbranche) konzeptionell durchgeführt werden kann. Hierzu erfolgt zunächst eine kursorische Darstellung der strategischen Rahmenbedingungen der Verlagsbranche. Anschliessend wird die Bildung einer Gruppe vergleichbarer Unternehmen begründet, bevor schliesslich der Begriff „Werttreiber“ im konkreten Branchenkontext definiert wird. Darauf aufbauend wird die Ableitung von Werttreibern und deren Integration in einem Finanzmodell gezeigt. In Abschnitt 3 erfolgt die Darstellung, wie eine stochastische Risikoanalyse schrittweise durchgeführt werden kann. Die Ergebnisse werden in Abschnitt 4 für eine Unternehmensbewertung inklusive der Darstellung des Mehrgewinns der gewählten Vorgehensweise sowie Sensitivitätsbetrachtungen genutzt. Die Arbeit schliesst mit einer Zusammenfassung und kritischen Würdigung der Ergebnisse.

1 Da beide Vorgehensweisen ähnlich klingen, sei darauf verwiesen, dass bei der Sicherheitsäquivalentmethode zuerst die Sicherheitsäquivalente der stochastischen Cash Flows und danach das Bewertungsergebnis als Kapitalwert der Sicherheitsäquivalente bestimmt wird. Bei der Risikoanalyse ist es umgekehrt. Zuerst wird der stochastische Kapitalwert bestimmt und danach dessen Sicherheitsäquivalent. Dies hat den Vorteil, dass intertemporale Abhängigkeiten vollständig in der Verteilung des stochastischen Kapitalwerts erhalten bleiben, vgl. dazu Bamberg et al. (2004, 101-118). 


\section{Identifikation von Werttreibern am Beispiel von Zeitungs- und Zeitschriftenverlagen}

\subsection{Rahmenbedingungen der Branche}

Die Durchführung einer Unternehmensbewertung mittels Planung und Prognose der bewertungsrelevanten finanziellen Überschüsse auf Basis von kausal und zeitlich vorgelagerten Werttreibern erfordert grundsätzlich ein tiefgehendes Verständnis für die Ausgangssituation und das Branchenumfeld, in dem das Bewertungsobjekt agiert (Drukarczyk/Ernst 2007, VIII-IX). Die zentralen strategischen Herausforderungen für Verlage können wie folgt zusammengefasst werden (Förster/Rhode 2009): Neue Wettbewerber in Form des Internets sowie die etablierten Mediengattungen TV, Radio und Kino adressieren ähnliche Informations- und Unterhaltungsbedürfnisse und bedrängen verstärkt das Kernprodukt „Print“. Aufgrund der zunehmenden multimedialen Konkurrenz ist damit zu rechnen, dass sich der Rückgang der Druckauflage, der bereits seit mehr als zehn Jahren zu beobachten ist, langfristig fortsetzen wird. Auch für den Rückgang der Werbeumsätze in Zeitungen und Zeitschriften scheint vorerst kein Ende in Sicht. Dieser resultiert im Wesentlichen aus der sinkenden Reichweite von Zeitungen und Zeitschriften sowie dem aktuell rezessiven Werbeumfeld. Zudem erhöht die geringe Zahlungsbereitschaft der Konsumenten für aktuelle Informationen und Unterhaltung die Abhängigkeit von Werbeeinnahmen. Die Wettbewerbsintensität wird sich im gesättigten und rückgängigen Marktumfeld daher tendenziell verstärken. Vor diesem Hintergrund reagieren die Verlage mit Anpassung der Kosten- und Erlösstrukturen im klassischen Kerngeschäft sowie mit der Erschliessung neuer digitaler und multimedialer Umsatzpotentiale. Zudem verzeichneten die Verlage in den letzten Jahren verstärkte M\&A-Aktivitäten. ${ }^{2}$

\subsection{Bildung einer Vergleichsgruppe}

Für die Bewertung eines nicht-börsennotierten Verlags im Kontext eines Unternehmenskaufs oder -verkaufs stellt sich zunächst die Frage nach geeigneten Vergleichsunternehmen (Richter 2005, 5). Eine Gruppe von Vergleichsunternehmen ist insbesondere im Falle der Eigenkapitalkostenbestimmung eines nicht-börsennotierten Unternehmens bedeutsam (Kruschwitz/Löffler 2008, 808). Die Eigenkapitalkosten des fiktiven nicht börsennotierten Unternehmens werden unter Verwendung des Capital Asset Pricing Models (CAPM) bei der Gruppe der Vergleichsunternehmen bestimmt und auf das Bewertungsobjekt übertragen. ${ }^{3}$ Darüber hinaus gilt es, die zukunftsorientierten Bewertungsüberlegungen durch Vergleichswerte in Form von Multiplikatoren zu plausibilisieren (Ballwieser 2007, 198 ff.; Coenenberg/Schultze 2002, 697-703). Beide Vorgehensweisen tragen dem Grundgedanken „Bewerten heisst Vergleichen“ Rechnung (Perridon/Steiner 2006, 493-500).

Die weiteren Ausführungen dieses Beitrags greifen den Gedanken auf und gehen einen Schritt weiter. Neben dem Rückgriff auf vergleichbare Unternehmen zur Eigenkapitalkos-

2 Seit 2005 wurden in der Branche pro Jahr weltweit mehr als 500 M\&A-Transaktionen (Zusammenschlüsse, Übernahmen, Minderheitsbeteiligungen, Joint Ventures) angekündigt, Thomson One Banker, 30.04.2009.

3 Für den Prozess der Übertragung wird für jedes Vergleichsunternehmen aus dem regressierten Beta ein „unlevered“ Beta auf Basis des aktuellen Verschuldungsgrads errechnet. Aus diesen Einzelergebnissen wird ein „Business Risk Index“ (BRI) mittels einer Gewichtung anhand des Bestimmtheitsmasses der Betaregressionen abgeleitet. Der so ermittelte BRI wird mit der unterstellten Zielkapitalstruktur „relevered“. Sowohl bei der Berechnung des „unlevered“ als auch des relevered“ Beta werden Grenzsteuersätze verwendet. 
tenbestimmung und zur Ableitung von Multiplikatoren wird deren historische finanzielle Performance herangezogen, um für eine Unternehmensbewertung unter Nutzung von stochastischen Risikoanalysen die erforderlichen Verteilungsannahmen der bewertungsrelevanten finanziellen Überschüsse abzuleiten (ähnlich Damodaran 2009, 18 f.).

Für das Fallbeispiel wird eine Vergleichsgruppe von 35 internationalen, börsennotierten Verlagen gebildet. Die Vergleichsunternehmen entstammen einer Grundgesamtheit von mehr als 240 börsennotierten Verlagen. ${ }^{4}$ Neben möglichst vergleichbaren Kerngeschäftsaktivitäten wird ein Mindestumsatz von 350 Mio. $€$ in 2007 als Auswahlkriterium festgelegt. Die berücksichtigten Unternehmen erwirtschaften den Grossteil ihres Umsatzes in den gesättigten Zeitungs- und Zeitschriftenmärkten Nordamerikas und Westeuropas. ${ }^{5}$

\subsection{Definition und Erhebung der Werttreiber}

Zur Identifikation der zu simulierenden Grössen wird auf das Shareholder-Value-Konzept von Rappaport (1998) zurückgegriffen. Das Konzept schlägt eine Brücke zwischen Unternehmenszielen, Strategie und Finanzplanung, indem es die Unternehmensbewertung mit Instrumenten der strategischen Planung verbindet (Schultze 2003, 236-239). Im Kern sieht es vor, die Wertkomponenten Free Cash Flow, Kapitalisierungszinssatz und Verschuldungsgrad dadurch zu operationalisieren, dass deren vorgelagerte Grössen (Umsatzwachstum, Gewinnmarge, etc.) auf den Managementebenen für Operationen, Investitionen und Finanzierung konkretisiert und durch geeignete Massnahmen gezielt beeinflusst werden. Abbildung 1 (Rappaport 1998, 56) veranschaulicht diesen Zusammenhang.

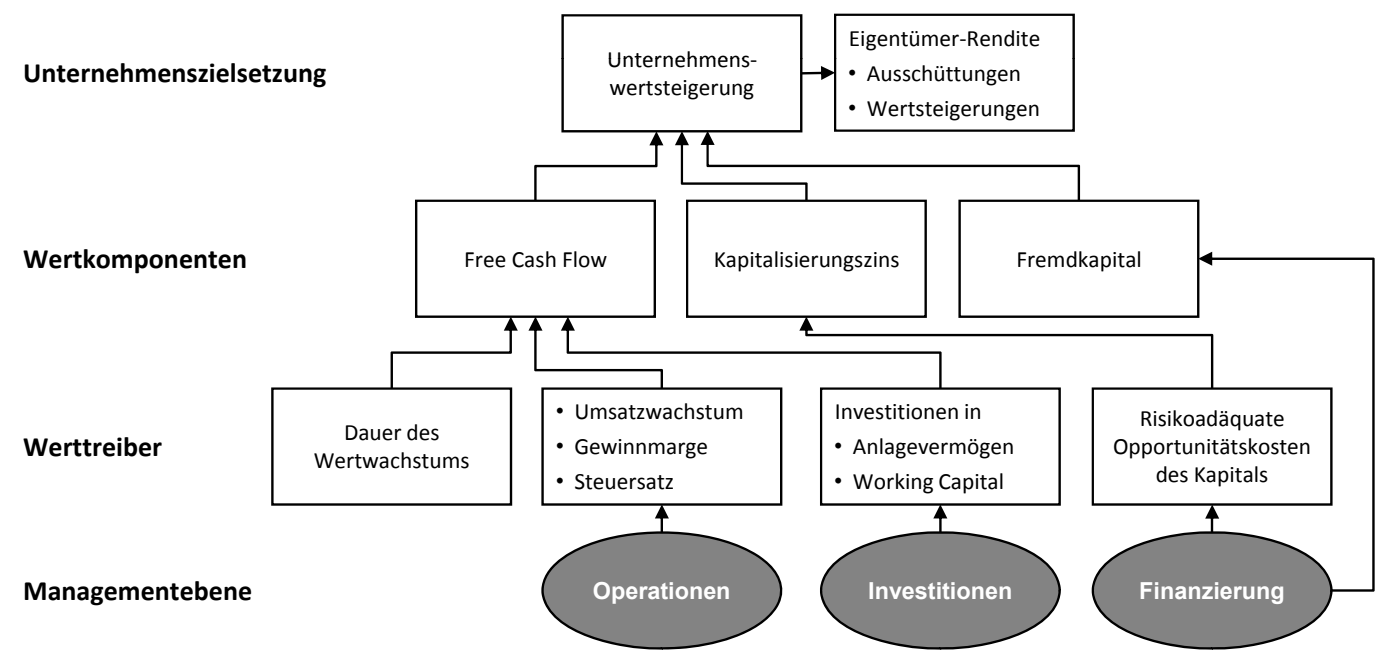

Abbildung 1: Werttreiber im Shareholder-Value-Konzept

Die vorgelagerten Grössen werden als Werttreiber (Value Driver) bezeichnet (Rappaport 1998, 55 f.). Diese bieten sich als Anknüpfungspunkte für Simulationen zur Durchführung

4 Global Industry Classification Standard 25401040 - Publishing, Thomson One Banker, 30.04.2009.

5 Der Autor ist sich der Vereinfachung bewusst, die dieser Vorgehensweise innewohnt. Vgl. hierzu kritisch Ballwieser (2007, 199 f.) und Kruschwitz/Löffler (2008, 808). 
einer Unternehmensbewertung auf Basis von Risikoanalysen an (Madrian/Auerbach 2009, 92 ff.; Keiber et al. 2002, 735-764; Schwartz/Moon 2000, 62-75). Grundsätzlich ist es auch denkbar, die Werttreiber weiter als in Abbildung 1 angedacht aufzuschlüsseln, z. B. durch Dekomposition des Umsatzwachstums in die zugrundeliegenden Preis- und Mengenkomponenten (Koller et al. 2005, 422 ff.; Rappaport 1998, 171-175). Da sich ein optimales Mass an Komplexitätsreduktion für die Modellbildung in einem solchen Kontext theoretisch kaum begründen lässt (Ballwieser 1990, VIII und $30 \mathrm{f}$.), wird hier auf eine weitere Aufteilung der Werttreiber verzichtet. Stattdessen werden die Werttreiber im Sinne des originären Shareholder-Value-Konzepts mittels „Percentage-of-Sales-Methode“ modelliert. Dies bietet sich auch insofern an, als dass die erforderlichen Vergangenheitsdaten für die börsennotierten Unternehmen aus der Vergleichsgruppe relativ problemlos in standardisierter Form über spezialisierte Finanzdatenanbieter bezogen werden können. ${ }^{6}$

Im Einzelnen werden acht Werttreiber genutzt, die es ermöglichen, das klassische PrintGeschäftsmodell sowie potentielle neue Geschäftsmodelle und Geschäftsfelder zu erfassen. Um die Werttreiber zukunftsgerichtet zu simulieren, müssen sie zunächst für die Vergleichsunternehmen definiert und aus den Vergangenheitsdaten erhoben werden, bevor sie in einer integrierten Finanzplanung zusammengeführt werden.

Wichtigster Werttreiber nach der „Percentage-of-Sales-Methode“ ist das zukünftige Umsatzwachstum. Im klassischen Print-Geschäft wird dieses primär durch Vertriebs- und Anzeigenerlöse bestimmt. Zentraler Mengentreiber beider Erlösquellen ist die Verkaufsauflage einzelner Titel. ${ }^{7}$ Die seit Jahren rückläufigen Auflagen und zuletzt gesunkenen Werbeerlöse deuten einen Rückgang der organischen Umsatzquellen des klassischen Print-Geschäfts an. Daher versuchen Verlage verstärkt, zusätzliches Wachstum durch M\&A und durch den Aufbau neuer (digitaler) Geschäftsmodelle zu generieren. Um sowohl organische als auch anorganische Wachstumseffekte zu erfassen, wird das historische Umsatzwachstum je Vergleichsunternehmen in aggregierter Form auf Basis der erzielten Umsatzerlöse für Periode $t$ und $t$-1erhoben. Für jedes Vergleichsunternehmen gilt:

$$
g_{\mathrm{t}}=\frac{\text { Sales }_{\mathrm{t}}}{\text { Sales }_{\mathrm{t}-1}}-1
$$

Als zweiter Werttreiber werden die betrieblichen Kosten OPEX exklusive Abschreibungen in einer aggregierten Position aus den Herstellkosten des Umsatzes COGS $_{t}$ und den Vertriebs- und Verwaltungskosten $S G A_{t}$ als Prozentgrösse vom Umsatz $c_{t}^{O P E X}$ für die Vergleichsgruppe definiert. Die $\operatorname{COGS}_{\mathrm{t}}$ werden bereinigt um die historischen Amortisationsbeträge für Rechte und Lizenzen $A_{\mathrm{t}}^{\mathrm{RL}}$ sowie Goodwill $A_{\mathrm{t}}^{\mathrm{GW}}$ und Abschreibungen $D_{\mathrm{t}}^{\mathrm{PPE}}$, um diese im Zuge der späteren Simulation separat adressieren zu können. Wesentliche Bestandteile der betrieblichen Kosten sind Materialkosten für Papier, Aufwendungen für be-

6 Hier ist z. B. Thomson ONE Banker zu nennen.

7 Zum einen ist die verkaufte Stückzahl über Einzelverkauf und Abonnements ausschlaggebend für die Vertriebserlöse. Zum anderen dient sie als messbarer Indikator für die Reichweite und Werbewirkung einzelner Titel. Damit ist sie zentraler Anhaltspunkt für die erzielbaren Anzeigenmengen und -preise. Diese lassen sich wiederrum messen als Werbefläche pro Ausgabe und über den sog. Tausender-Kontakt-Preis. Der Tausender-Kontakt Preis (TKP) gibt an, welcher Geldbetrag eingesetzt werden muss, um 1.000 Personen einer Zielgruppe zu erreichen. 
zogene Fremddrucke und Honorare für externe Publisher sowie Personalkosten für Angestellte und Redakteure. Somit gilt für die Quote der operativen Kosten $c_{t}^{\text {OPEX }}$ für die Vergleichsunternehmen:

$$
c_{t}^{\mathrm{OPEX}}=\frac{\operatorname{COGS}_{\mathrm{t}}+S G A_{t}}{\text { Sales }_{t}}
$$

Die Investitionsraten (IR) für Rechte und Lizenzen (RL), Firmenwert (GW) und Sachanlagen (PPE) bilden weitere Werttreiber, die auf Basis der Vergleichsgruppe erhoben werden. Die Ermittlung erfolgt separat für jedes Unternehmen jeweils auf Basis der jährlichen Investitionsbeträge (CAPEX) in Prozent der aggregierten Umsatzerlöse:

$$
\begin{aligned}
I R_{t}^{R L} & =\frac{\text { CAPEX }_{t}^{R L}}{\text { Sales }_{t}} \\
I R_{t}^{G W} & =\frac{\text { CAPEX }_{t}^{G W}}{\text { Sales }_{t}} \\
I R_{t}^{P P E} & =\frac{\text { CAPEX }_{t}^{P P E}}{\text { Sales }_{t}}
\end{aligned}
$$

Die jährlichen Investitionsbeträge $C A P E X_{t}^{R L}, C A P E X_{t}^{G W}, C A P E X_{t}^{P P E}$ werden vereinfacht als jährlicher Differenzbetrag der zugehörigen Bilanzpositionen für Rechte und Lizenzen, Firmenwert und Sachanlagen zuzüglich der jährlichen Amortisations- und Abschreibungsbeträge $A_{\mathrm{t}}^{\mathrm{RL}}, A_{\mathrm{t}}^{\mathrm{GW}}$ bzw. $D_{\mathrm{t}}^{\mathrm{PPE}}$ errechnet.

Als weitere Werttreiber werden die Hauptbestandteile des Working Capital für die Vergleichsunternehmen erhoben. Hierbei wird zwischen der Umschlagsdauer der Vorräte in Tagen $I N V D_{t}$ sowie zwischen den durchschnittlichen Zahlungszielen für Forderungen $R E C D_{t}$ und Verbindlichkeiten $P A Y D_{t}$ in Tagen differenziert:

$$
\begin{aligned}
& {I N V D_{t}=}_{\text {Inventory }_{t}}^{\text {COGS }_{t}} \times 365 \\
& \text { RECD }_{t}=\frac{\text { Accounts Receivable }_{t}}{\text { Sales }_{t}} \times 365 \\
& \text { PAYD }_{t}=\frac{\text { Accounts Payable }_{t}}{\text { COGS }_{t}} \times 365
\end{aligned}
$$

Tabelle 1 zeigt die Ausprägungen der acht identifizierten und erhobenen Werttreiber exemplarisch für das Jahr 2007 für die 35 Vergleichsunternehmen. Sie erklären ca. 80\% der Kostenstruktur und mehr als $60 \%$ der Bilanzsumme eines durchschnittlichen Verlags. Das bedeutet, dass die Werttreiber entsprechend der unterstellten Berechnungslogik den überwiegenden Anteil der beobachteten Grössen zu erklären vermögen. 


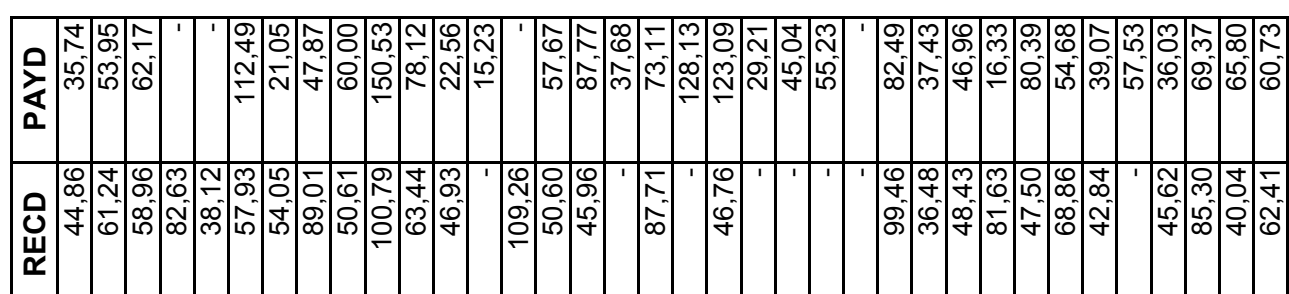

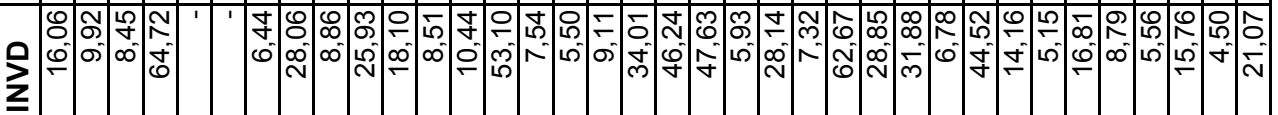

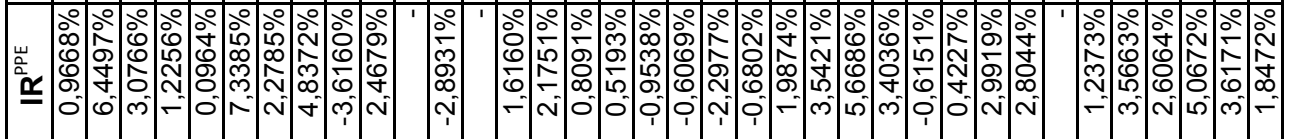

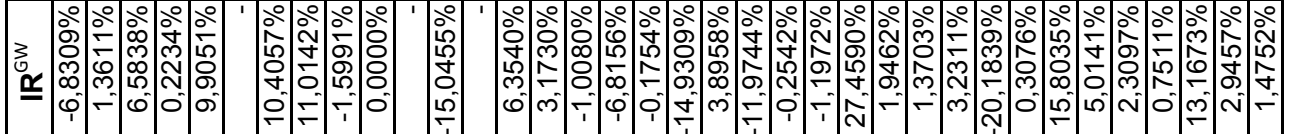

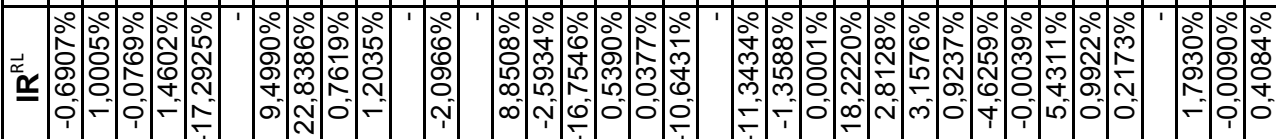

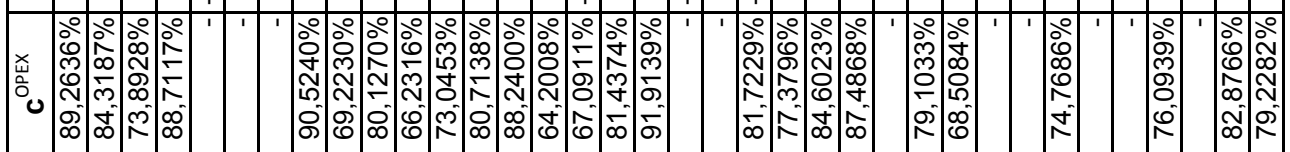

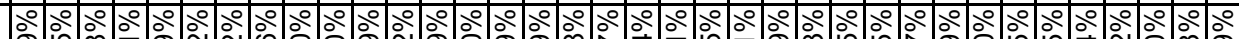

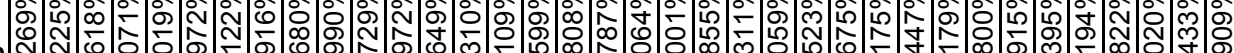

凹

-

బิळ

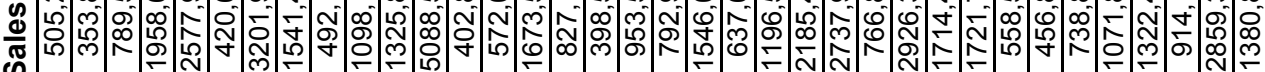

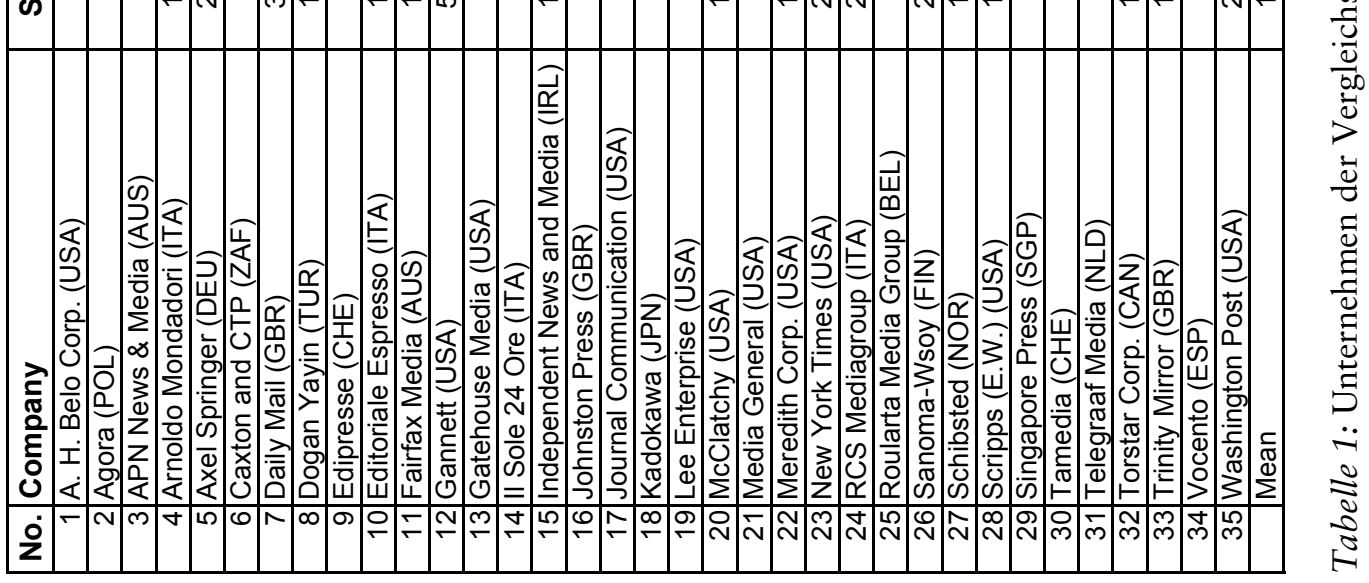

8 Thomson One Banker, 30.04.2009. Die fehlenden Datenpunkte waren entweder nicht verfügbar oder wurden aufgrund von offensichtlichen Eingabefehlern in der Datenbank nicht einbezogen. 
In den nachfolgenden Ausführungen wird vereinfachend unterstellt, dass die erhobenen Werttreiberdaten der Vergleichsunternehmen auf das Geschäftsmodell eines spezifischen Verlags als Bewertungsobjekt übertragen werden können. In der Bewertungspraxis stellt dies in der Regel aber eine Herausforderung dar, denn die Auswahl der Vergleichsunternehmen ist neben dem grundsätzlichen Problem der Verfügbarkeit von Daten auch immer von den Interessen der beteiligten Parteien getrieben. Dies gilt im Besonderen im Kontext von Transaktionen, wenn Bewertungsergebnisse in Preisüberlegungen münden. Unter der Prämisse einer Übertragbarkeit lassen sich die Vergangenheitsdaten der Vergleichsunternehmen nutzen, um daraus Verteilungen für die bewertungsrelevanten finanziellen Überschüsse des Bewertungsobjekts abzuleiten.

\subsection{Zusammenführung der Werttreiber in eine integrierte Finanzplanung}

Die formale Zusammenführung der identifizierten Werttreiber erfolgt in einer integrierten Finanzplanung (vgl. zu den folgenden Ausführungen Koller et al. 2005, 240-257; IDW 2008, 23). Diese besteht aus Gewinn- und Verlustrechnung (GuV), Bilanz, Kapitalflussrechnung und einer vereinfachten Gewinnverwendungsrechnung. Sie wird modellgestützt unter Nutzung des Tabellenkalkulationsprogramms Microsoft ${ }^{\circledR}$ Excel erstellt. Um zukünftige Verteilungen für die bewertungsrelevanten finanziellen Überschüsse des Bewertungsobjekts zu schätzen, ist es notwendig, die integrierte Finanzplanung so zu modellieren, dass die finanziellen Plangrössen auf Basis von Verteilungsannahmen mittels Monte-CarloSimulation generiert werden können. Die erforderlichen Verteilungsannahmen werden unter Rückgriff auf die erhobenen Werttreiberdaten der Vergleichsunternehmen abgeleitet. Darüber hinaus sind auch solche Grössen in der Finanzplanung abzubilden, die nicht über die identifizierten Werttreiber adressiert werden, wie z. B. Eigenkapital und liquide Mittel. Beide Grössen werden innerhalb der integrierten Finanzplanung errechnet. Ausserdem bedarf es für jede Planperiode einen modellgestützten Ausgleichsmechanismus, der die Integrität der Rechenwerke im Falle von Unter- oder Überdeckungen an finanziellen Mitteln periodisch sicherstellt.

\subsubsection{Gewinn- und Verlustrechnung}

Die Umsatzerlöse pro Plan-Periode $t$ für das Bewertungsobjekt ergeben sich durch Fortschreibung der Umsatzerlöse aus der Vorperiode multipliziert mit dem Wachstumsfaktor $\left(1+g_{t}\right)$. Somit gilt für das Bewertungsobjekt:

$$
\text { Sales }_{t}=\text { Sales }_{t-1} \times\left(1+g_{t}\right)
$$

Die Wachstumsrate $g_{t}$ wird zunächst durch Bildung eines Durchschnittswerts (Mean) für die Wachstumsraten der Vergleichsunternehmen aus Tabelle 1 geschätzt. In einem zweiten Schritt erfolgt im Rahmen der Risikoanalyse die Schätzung der Wachstumsrate mittels Monte-Carlo-Simulation auf Basis von geschätzten historischen Verteilungen für die erhobenen Werttreiberdaten der Vergleichsunternehmen (siehe dazu Abschnitt 3).

Die operativen Kosten OPEX als Summe aus Umsatzkosten COGS $_{t}$ sowie Vertriebs- und Verwaltungskosten $S G A_{t}$ sind aggregiert als prozentualer Anteil $c_{t}^{O P E X}$ der zukünftigen Umsatzerlöse dargestellt. Der operativen Kostenquote liegt die entsprechende historische Verteilung der Vergleichsdaten zugrunde. Folglich ist auf übereinstimmend aufbereitete 
historische Kostendaten für das Bewertungsobjekt und die Vergleichsunternehmen zu achten, um Inkonsistenzen in der Datenbasis zu vermeiden. Ist dies sichergestellt, kann die Ermittlung der gesamten operativen Kosten erfolgen und eine proportionale Aufteilung in die GuV-Positionen COGS $_{t}$ und $S G A_{t}$ wird über einen konstanten Faktor $\alpha$ vorgenommen. Im Fallbeispiel sind die simulierten operativen Kosten im Verhältnis $70 \%$ zu $30 \%$ aufgeteilt.

$$
\begin{aligned}
& \text { COGS }_{t}=\text { Sales }_{t} \times c_{t}^{\text {OPEX }} \times \alpha \\
& \text { SGA }_{t}=\text { Sales }_{t} \times c_{t}^{\text {OPEX }} \times(1-\alpha)
\end{aligned}
$$

Die GuV-Position other operating income wird unverändert als absolute Grösse aus der Historie fortgeschrieben. Die Positionen other operating expenses und finance income entwickeln sich über eine konstante historische Rate proportional zu den geschätzten Umsatzerlösen. ${ }^{9}$

Der zukünftige Steueraufwand (tax expense) ergibt sich vereinfacht durch Multiplikation der GuV-Zwischengrösse earnings before tax (EBT) als Bemessungsgrundlage mit einem integrierten Ertragsteuersatz $s_{t}$. Ist der EBT einer Periode negativ, wird für diese Periode eine Steuerbelastung von Null unterstellt. Verlustvorträge werden nicht berücksichtigt. Im deutschen Steuerregime unter Annahme eines Hebesatzes $h$ von $400 \%$ bei einer heute einheitlichen Steuermesszahl $m$ von 3,5\% beträgt die Gewerbeertragsteuerquote $14 \%$. Gemäss $\$ 23 \mathrm{KStG}$ ist seit 2008 ein Körperschaftsteuersatz $s_{K S t}$ von $15 \%$ plus Solidaritätszuschlag $s_{S o l Z}$ in Höhe von 5,5\% zu verwenden. Somit ergibt sich im Fallbeispiel ein integrierter Ertragssteuersatz von 29,825\%. Dieser wird wie folgt bestimmt:

$$
s_{t}=s_{K S t} \times\left(1+s_{\text {SolZ }}\right)+s_{\text {GewSt }}=s_{K S t} \times\left(1+s_{\text {SolZ }}\right)+m \times h
$$

Tabelle 2 zeigt die geplante Entwicklung der GuV für das Bewertungsobjekt auf Basis der Durchschnittswerte (Mean) für die Werttreiber aus Tabelle 1.

\subsubsection{Bilanz}

Analog zur GuV werden die erhobenen Werttreiberdaten der Vergleichsunternehmen auf die entsprechenden Bilanzpositionen des Bewertungsobjekts übertragen. Zunächst gilt es, die periodischen Investitionsausgaben (CAPEX) für Rechte und Lizenzen (RL), Geschäftsund Firmenwert (GW) sowie Sachanlagen (PPE) unter Rückgriff auf die Verteilungen für die jeweiligen Investitionsraten (IR) der Vergleichsunternehmen bezogen auf die Umsatzerlöse des Bewertungsobjekts abzuleiten:

9 Die Verhältniszahl der „other operating expenses“ beträgt annahmegemäss 5,12\% vom Umsatz. Das „finance income“ steht auch im Verhältnis zum Umsatz. Der angenommene Prozentsatz von 1,28 in 2010 reduziert sich in den Planjahren jede Periode um 0,02 Prozentpunkte. Das Jahr 2009 stellt insofern eine planerische Ausnahme dar, als dass es keinen Zinsertrag / -verlust aus der rechnerischen Bilanzausgleichsposition gibt. Diese Grösse hat in 2009 aufgrund der ausgeglichenen Ist-Bilanz keine Bedeutung. 


$$
\begin{aligned}
& \text { CAPEX }_{t}^{R L}=\text { Sales }_{t} \times I R_{t}^{R L} \\
& \text { CAPEX }_{t}^{G W}=\text { Sales }_{t} \times I R_{t}^{G W} \\
& \text { CAPEX }_{t}^{P P E}=\text { Sales }_{t} \times I R_{t}^{P P E}
\end{aligned}
$$

Um die periodische Entwicklung der zugehörigen Bilanzpositionen des Anlagevermögens ermitteln zu können, werden vereinfacht die durchschnittlichen historischen Amortisations- und Abschreibungsquoten in Prozent des Vorjahresbestands der jeweiligen Aktivposition herangezogen. Im Fallbeispiel werden auf diese Weise konstante Amortisations- und Abschreibungsquoten für Rechte und Lizenzen, Geschäfts- und Firmenwert sowie Sachanlagen in Höhe von 1,1\%, 0,5\% und 1,8\% des aktuellen Umsatzes unterstellt, um die periodischen Amortisations- und Abschreibungsbeträge $A_{t}^{R L}, A_{t}^{G W}$ und $D_{t}^{P P E}$ zu schätzen. Zudem gilt für Positionen des Anlagevermögens die Nebenbedingung, dass diese nicht negativ werden dürfen, falls es in einzelnen Simulationsdurchläufen zu hohen negativen Investitionsraten in Prozent der Umsatzerlöse gleichbedeutend mit Desinvestitionen kommt. Folgende Gleichungen beschreiben die simulierte Entwicklung der Positionen des Anlagevermögens einschliesslich der Nichtnegativitätsbedingung:

$$
\begin{aligned}
& R L_{t}=\left\{\begin{array}{l}
R{ }_{t-1}+\text { CAPEX }_{t}^{R L}-A_{t}^{R L}, \text { falls } R L{ }_{t-1}+\text { CAPEX }_{t}^{R L}-A_{t}^{R L}>0 \\
0, \text { sonst }
\end{array}\right. \\
& G W_{t}=\left\{\begin{array}{l}
G W_{t-1}+\text { CAPEX }_{t}^{G W}-A_{t}^{G W}, \text { falls } G W_{t-1}+\text { CAPEX }_{t}^{G W}-A_{t}^{G W}>0 \\
0, \text { sonst }
\end{array}\right. \\
& P P E_{t}=\left\{\begin{array}{l}
P P E_{t-1}+\text { CAPEX }_{t}^{P P E}-D_{t}^{P P E}, \text { falls PPE } \\
0, \text { sonst }
\end{array}\right.
\end{aligned}
$$

Die periodische Fortschreibung der Hauptpositionen des Working Capital für das Bewertungsobjekt erfolgt auf Basis der Umsatzerlöse Sales ${ }_{t}$, Umsatzkosten COGS $_{t}$ und den geschätzten Umschlagsdauern $I N V D_{t}, R E C D_{t}$ und $P A Y D_{t}$ der Vergleichsgruppe.

$$
\begin{aligned}
& \text { Inventory }_{t}=\text { COGS }_{t} \times \text { INVD }_{t} \times \frac{1}{365} \\
& \text { Accounts Receivable }_{t}=\text { Sales }_{t} \times \text { RECD }_{t} \times \frac{1}{365} \\
& \text { Accounts Payable }_{t}=\text { COGS }_{t} \times \text { PAYD }_{t} \times \frac{1}{365}
\end{aligned}
$$

Die Tabellen 3 und 4 zeigen die geplante Entwicklung der Aktiva und Passiva für das Bewertungsobjekt auf Basis der Durchschnittswerte (Mean) für die Werttreiber aus Tabelle 1. 


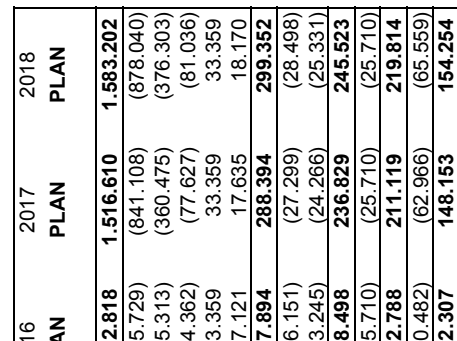

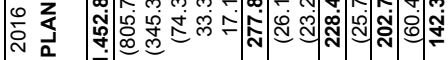

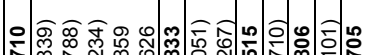

管

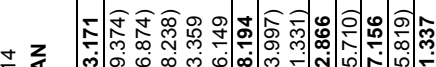

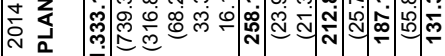

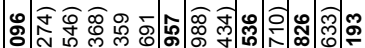

茫

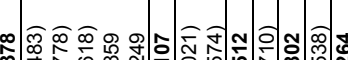

范

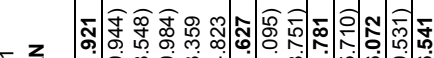

产 至

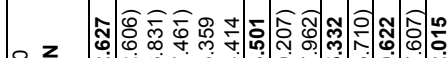

家 去

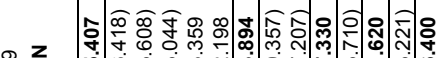

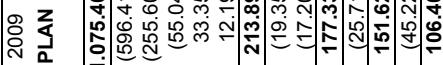

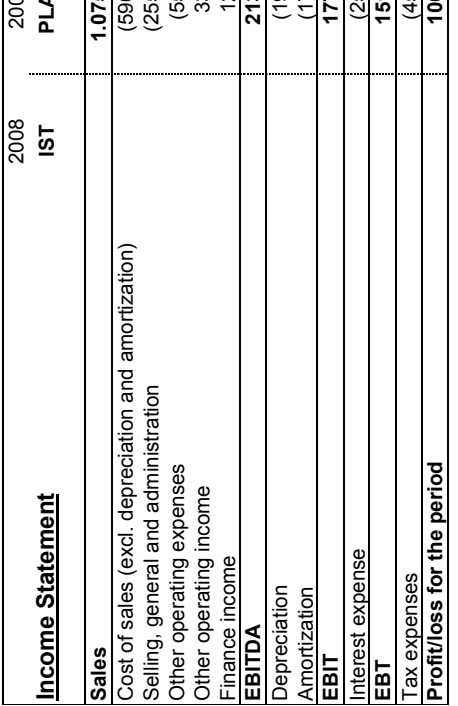

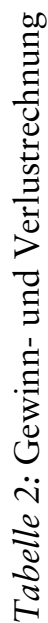

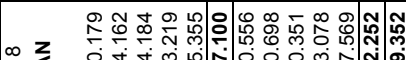

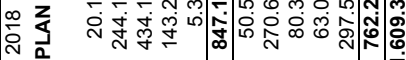

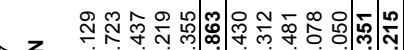

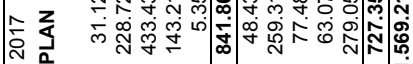

—

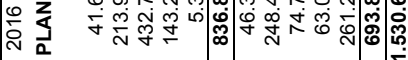

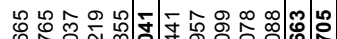

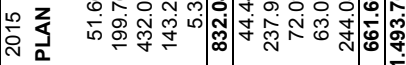

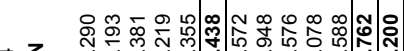

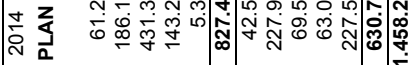

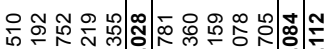

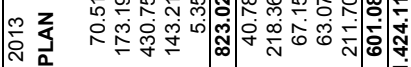

舟

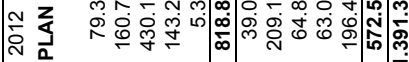

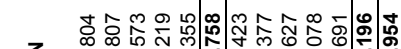

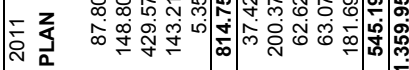

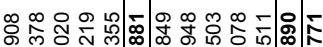

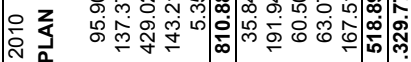

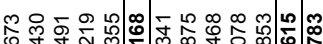

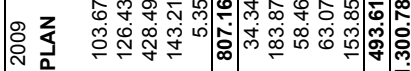

L

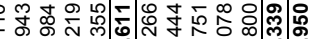

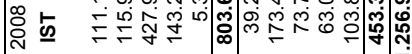

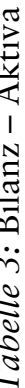




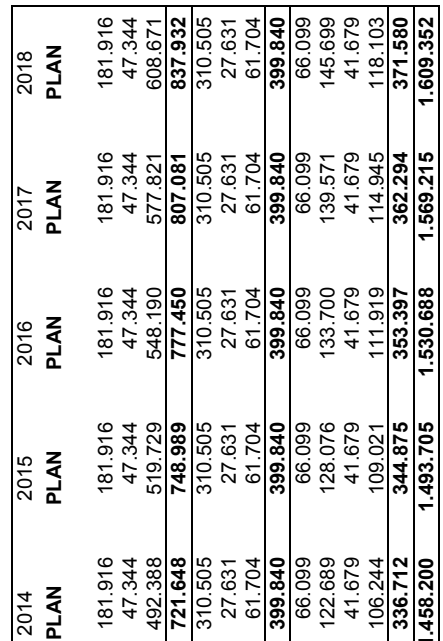

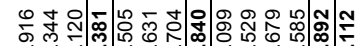

家

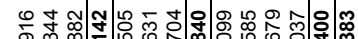

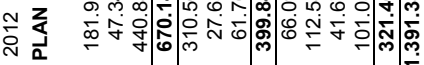

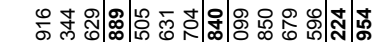

究告

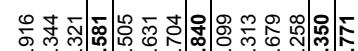

定 毛

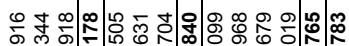

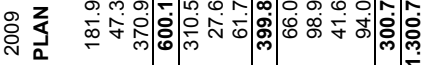

ñ

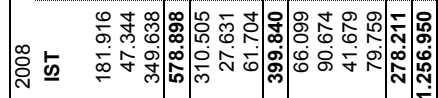

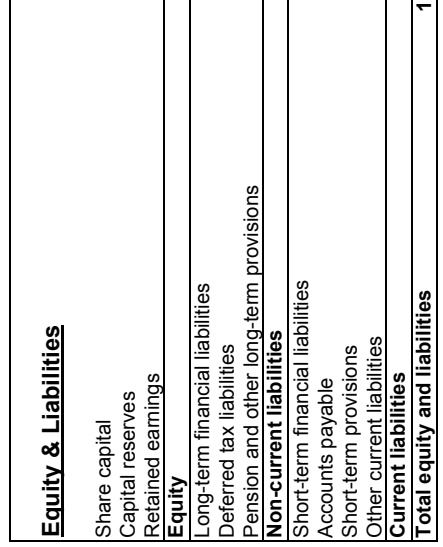

Die Unternehmung, 65. Jg., 4/2011

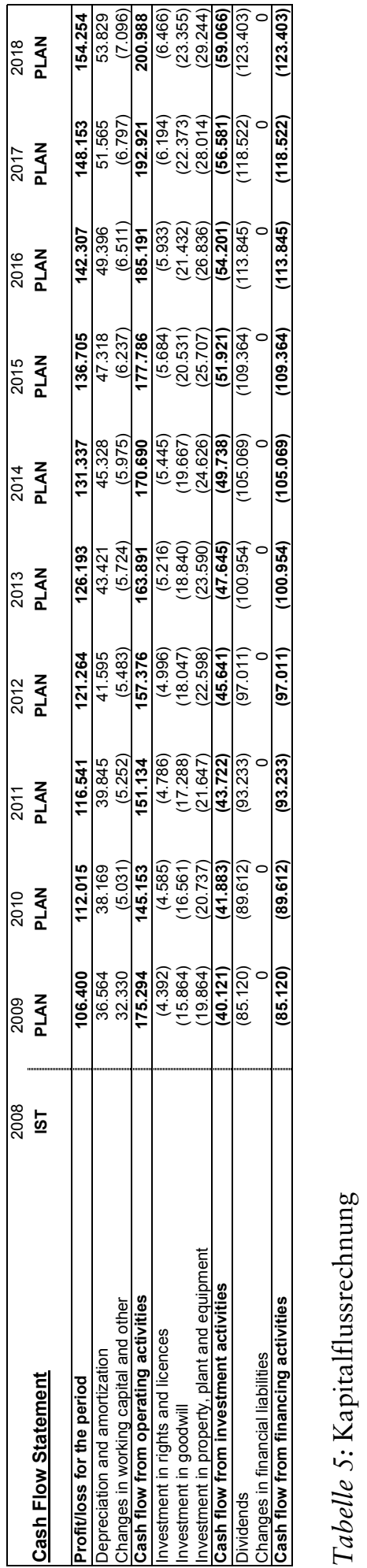

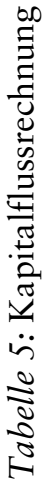


Alle weiteren Bilanzpositionen des Fallbeispiels werden entweder unverändert fortgeschrieben (long-term investments, deferred tax assets, short-term investments, long-term financial liabilities, deferred tax liabilities, pension and other long-term provisions, short-term provisions) oder über einen konstanten Prozentwert in Abhängigkeit der Umsatzentwicklung modelliert (other current liabilities und other current assets). ${ }^{10}$ Die Eigenkapitalpositionen share capital und capital reserves werden konstant fortgeschrieben. Die Gewinnrücklagen (retained earnings) ergeben sich vereinfacht aus dem Bestand der Vorperiode zuzüglich / abzüglich des ausschüttbaren Gewinns / Verlusts der Periode abzüglich der ausgezahlten Dividenden. Diese wird im Fallbeispiel annahmegemäss über eine konstante Ausschüttungsquote in Höhe von $80 \%$ der Gewinne als Schätzer für eine branchenübliche Quote errechnet.

Die modellierte Finanzplanung ist zudem so aufgebaut, dass die Gleichheit von Aktiva $A S_{t}$ und Passiva als Summe aus Eigenkapital $E_{t}$ und Fremdkapital $L_{t}$ für alle Simulationsdurchläufe stets eingehalten wird. Der Bestand an liquiden Mitteln Cash $_{t}$ und die kurzfristigen Finanzverbindlichkeiten $S T L_{t}$ fungieren hierzu als Ausgleichspositionen. Sollte die Simulation der Werttreiber dazu führen, dass in einer Periode Zahlungsmittelüberschüsse generiert werden, erfolgt automatisch eine Erhöhung der liquiden Mittel in entsprechender Höhe. Es gilt:

$$
\text { Cash }_{t}=\left\{\begin{array}{l}
E_{t}+L_{t}-A S_{t}^{\text {excluding Cash }}, \text { falls } E_{t}+L_{t}>A S_{t}^{\text {excluding Cash }} \\
\text { Cash }_{t}^{\text {mimimum }}, \text { sonst }
\end{array}\right.
$$

Im Komplementärfall, wenn in einer Periode Zahlungsmittelunterdeckung entsteht und die Aktiva die Passiva übersteigen, wird der Differenzbetrag über eine Erhöhung der kurzfristigen Finanzverbindlichkeiten ausgeglichen. Dann gilt:

$$
S T L L_{t}=\left\{\begin{array}{l}
A S_{t}-E_{t}-L_{t} \text { excluding } S T L \\
S T L \\
S_{t}^{\text {mimimum }}, \text { falls } E_{t}+L_{t}>A S_{t}^{\text {excluding } S T L}
\end{array}\right.
$$

Um zu hohe Liquiditäts- und Verschuldungsschwankungen zu vermeiden, sind im Fallbeispiel jeweils ein minimaler Kassenbestand Cash $_{t}^{\text {mimimum }}$ und eine Untergrenze für die Verbindlichkeiten $S T L \underset{t}{\text { mimimum }}$ festgelegt. Zudem wird ein konstanter Habenzinssatz in Höhe von 3,0\% und ein konstanter Fremdkapitalkostensatz von 6,81\% unterstellt.

\subsubsection{Kapitalflussrechnung}

Auf Basis der geplanten GuV und Bilanz werden die periodischen Accounting Cash Flows nach der indirekten Methode abgeleitet (siehe Tabelle 5). Die periodische Veränderung des Working Capital $\triangle W_{C A P}{ }_{t}$ wird aus der Differenz des Working Capital für Periode $t$ und $t-1$ bestimmt. Die Position Working Capital errechnet sich aus den simulierten oder fort-

10 Das Verhältnis zum Umsatz bei den „other current liabilities“ beträgt im ersten Planjahr annahmegemäss $8,74 \%$ und fällt danach um 0,17 Prozentpunkte, wobei sich die Reduzierung jedes Jahr um 0,01 Prozentpunkte verringert. Die „other current assets“ betragen annahmegemäss in der ersten Planperiode 5,44\% vom Umsatz und fallen jedes weitere Planjahr um 0,04 Prozentpunkte. 
geschriebenen Bilanzpositionen inventory, accounts receivable, other current assets, accounts payable und other current liabilities:

$$
W C A P_{t}=I N V_{t}+R E C_{t}+O C A_{t}-P A Y_{t}-O C L_{t}
$$

Die CAPEX $_{t}$ errechnen sich aus der Summe der Brutto-Investitionsbeträge für Rechte und Lizenzen, Firmenwert und Sachanlagen:

$$
\text { CAPEX }_{t}=\text { CAPEX }_{t}^{R L}+\text { CAPEX }_{t}^{G W}+\text { CAPEX }_{t}^{P P E}
$$

Die integrierte Finanzplanung bildet somit die Grundlage für die Ableitung der bewertungsrelevanten finanziellen Überschüsse und zur Durchführung von stochastischen Risikoanalysen bei der Unternehmensbewertung (Madrian/Auerbach 2009, 96 f.).

\section{Durchführung der Risikoanalyse}

\subsection{Vorgehensweise}

Im ersten Schritt gilt es, die relevanten finanziellen Grössen zu definieren, die als Zielgrössen simuliert werden (vgl. zur Vorgehensweise ähnlich Brealey et al. 2008, 78-283; Damodaran, 2009, 26; Perridon/Steiner 2006, 117-121). Danach wird gezeigt, wie Verteilungshypothesen für die identifizierten Werttreiber, die als Eingabegrössen für das Modell dienen, aus den erhobenen Daten gebildet werden können. Im dritten Schritt werden Korrelationen der Werttreiber identifiziert und im Modell erfasst. Im vierten Schritt erfolgt die eigentliche Durchführung der stochastischen Risikoanalyse mittels Monte-Carlo-Simulation, um Verteilungen für die bewertungsrelevanten finanziellen Überschüsse zu generieren.

\subsection{Definition der zu simulierenden Zielgrössen}

Ziel der Risikoanalyse ist es, periodische Verteilungen für die Free Cash Flows simulationsgestützt zu schätzen und daraus Erwartungswerte für die Unternehmensbewertung abzuleiten, die in einem weiteren Schritt erfolgt. Die Durchführung der Berechnungen erfolgt unter Nutzung des Software Add-Ins Crystal Ball ${ }^{11}$ für Microsoft ${ }^{\circledR}$ Excel. $^{12}$ Als bewertungsrelevante finanzielle Überschüsse werden unverschuldete Free Cash Flows $F C F_{t}^{u}$ entsprechend dem Weighted Average Cost of Capital-Ansatz der Discounted Cash Flow-Methode verwendet (DCF-WACC) (vgl. statt vieler Schultze 2003, 369-414). Diese ergeben sich auf Basis der oben beschriebenen integrierten Finanzplanung für jede Planperiode wie folgt:

$$
F C F_{t}^{u}=E B I T t_{t} \times\left(1-s_{t}\right)+D_{t}^{P P E}+A_{t}^{R L}+A_{t}^{G W}-C^{P A P E X} t_{t}-\Delta W C A P_{t}
$$

11 Crystal Ball ist ein eingetragenes Markenzeichen von Oracle ${ }^{\circledR}$.

12 Eine praxisorientierte Einführung in die Risikosimulation mit Hilfe der Simulationssoftware Crystal Ball bieten u. a. Grob/Mrzyk (1998, 120-129). Grundsätzlich eignen sich auch andere Add-Ins für Microsoft ${ }^{\circledR}$ Excel zur Durchführung von Monte Carlo Simulationen, wie z. B. @Risk. @Risk ist ein eingetragenes Markenzeichen von Palisade ${ }^{\circledR}$. 
Der EBIT ${ }_{t}$ errechnet sich wie folgt aus den Grössen Umsatz Sales ${ }_{t}$, operative Kosten $O P E X_{t}$, sonstige Aufwendungen $O E_{t}$, sonstige Erträge $O I_{t}$, finanzielle Erträge $F_{t}$, Abschreibungen auf Sachanlagen $D_{t}^{P P E}$, Amortisation von Rechten und Lizenzen $A_{t}^{R L}$ und Amortisation von Goodwill $A_{t}^{G W}$ :

$$
\text { EBIT }_{t}=\text { Sales }_{t}-\text { OPEX }_{t}-O E_{t}+O I_{t}+F I_{t}-D_{t}^{P P E}-A_{t}^{R L}-A_{t}^{G W}
$$

Die CAPEX $_{t}$ und die periodische Veränderung des Working Capital $\triangle W C A P_{t}$ werden wie in 2.4.3 beschrieben errechnet.

Da die Bewertung in der Praxis meist in einem Phasenmodell erfolgt (Rappaport 1998, 40 ff.; Schultze 2003, 240 f.; Koller et al. 2005, 275 ff.; IDW S1 2008, 16 Tz. 76-79), müssen zudem geeignete Anknüpfungspunkte zur Bestimmung des Fortführungswerts (Terminal Value) jenseits der Detailplanungsphase definiert werden. Dazu wird auf das sog. Value Driver Modell nach Koller et al. (2005, 274 ff.) zurückgegriffen. Durch Zusammenfassen der Summanden aus Gleichung 26 erhält man die operative Gewinngrösse $N^{N O P L A T}{ }_{t}$ bzw. durch Zusammenfassen der Subtrahenden die aggregierten Netto-Investitionen $\mathrm{NI}_{t}$ (Koller et al. 2005, 108-112):

$$
F C F_{t}^{u}=\operatorname{NOPLAT}_{t}+N I_{t}
$$

Dieser Zusammenhang lässt sich umformulieren zu:

$$
F C F_{t}^{u}=\operatorname{NOPLAT}_{t}\left(1-I R_{t}\right)
$$

Die jährliche Investitionsrate $I R_{t}$ lässt sich wiederum darstellen als Quotient aus der jährlichen nominalen Wachstumsrate $g_{t}^{N}$ der operativen Gewinngrösse NOPLAT $_{t}$ und der zu schätzenden Rendite auf das neu investierte Kapital ROIC $_{I}$ :

$$
I R_{t}=\frac{g_{t}^{N}}{R O I C_{I}} \Leftrightarrow g_{t}^{N}=I R_{t} \times \text { ROIC }_{I}
$$

Beide Grössen dienen als Anknüpfungspunkt zur Bestimmung des Fortführungswerts auf Basis einer nachhaltigen Wachstumsrate der operativen Gewinne $g_{t}^{N}$ und der Rendite

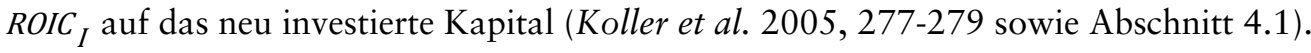

\subsection{Bildung von Verteilungshypothesen für die Eingabeparameter}

Um periodische Risikoprofile für die unverschuldeten Free Cash Flows zu generieren und daraus Erwartungswerte für die Bewertung abzuleiten, ist es erforderlich, die Wahrscheinlichkeitsverteilungen der simulierten Zufallsvariablen - in diesem Fall die identifizierten Werttreiber - zu schätzen (Brealey et al. 2008, 281; Perridon/Steiner 2006, 117). Zur Bildung von Verteilungshypothesen für die Werttreiber werden die erhobenen historischen Werttreiberdaten der Vergleichsunternehmen exemplarisch für das Jahr 2007 als Stichprobe herangezogen und im Weiteren dargestellt. Indes gilt es, besondere Aufmerksamkeit auf die Auswahl des bzw. der relevanten Jahre zu richten. Die jeweilige Datenbasis kann das 
Ergebnis nicht unmassgeblich beeinflussen. Dies zeigen auch die Sensitivitätsbetrachtungen in Abschnitt 4.2 zur Durchführung der Unternehmensbewertung.

Bei allen acht Werttreibern handelt es sich um stetige Zufallsvariablen, deren Wahrscheinlichkeitsverteilungen jeweils mit Hilfe von Crystal Ball geschätzt werden. Auf Basis der erhobenen Daten aus Tabelle 1 ergeben sich jeweils Wahrscheinlichkeitsverteilungen für die einzelnen Werttreiber, sog. Best-Fit Verteilungen. Die Best-Fit Verteilungen werden ermittelt, indem unter Berücksichtigung der Symmetrie der erhobenen Daten sowie der Wahrscheinlichkeit und der Lage von Ausreissern, über statistische Anpassungstests geprüft wird, ob die Charakteristiken der erhobenen Daten einer bestimmten Verteilung entsprechen (vgl. für eine Übersicht wichtiger Verteilungen Vose 1996, 51-92; Bamberg et al. 2007, 99-111; Rubinstein/Kroese 2008, 5; sowie zur hier verwendeten Vorgehensweise Vose 1996, 103-152). Dies erfolgt softwaregestützt auf Basis des Anderson-Darling Anpassungstests, einer Erweiterung des Kolmogorov-Smirnov-Anpassungstests (vgl. zu den genannten Anpassungstests ausführlicher Vose 1996, 126-132). Die Ergebnisse der geschätzten Verteilungen für die acht Werttreiber auf Basis des Jahres 2007 sind in Tabelle 6 übersichtsartig dargestellt.

Die Auswertungen zeigen, dass zur Schätzung der zukünftigen Umsatzwachstumsrate $g_{t}$ und der durchschnittlichen Zahlungsziele für Verbindlichkeiten $P A Y D_{t}$ im Fallbeispiel auf eine Max Extreme-Verteilung (Extremwertverteilung) zurückgegriffen wird. Diese ist allgemein durch Asymmetrie und überwiegend positive Ausreisser gekennzeichnet (Vose 1996, 68 f.). Für die operative Kostenquote $c_{t}^{O P E X}$ wird eine Min Extreme-Verteilung ermittelt, die ebenso durch Asymmetrie aber überwiegend negative Ausreisser gekennzeichnet ist (Vose 1996, 91 f.). Für die Investitionsraten $I R_{t}^{R L}$ für Rechte und Lizenzen, $I R_{t}^{G W}$ für Firmenwert und $I R_{t}^{P P E}$ für Sachanlagen wird jeweils eine Logistic-Verteilung (logistische Verteilung) unterstellt, die durch Symmetrie und geringe Wahrscheinlichkeit von Ausreissern gekennzeichnet ist (Vose 1996, 77 f.). Für die Umschlagsdauer der Vorräte $I N V D_{t}$ und das durchschnittliche Zahlungsziel für Forderungen $R E C D_{t}$ in Tagen wird von einer Lognormal-Verteilung ausgegangen, die durch Asymmetrie und überwiegend positive Ausreisser gekennzeichnet ist (Vose 1996, 79-81).

Die Anderson-Darling Teststatistik wird für jeden der Werttreiber errechnet. Im Allgemeinen gilt, je niedriger die Anderson-Darling Teststatistik, desto besser passt die geschätzte Verteilung zu den erhobenen Daten. Die ermittelten Testwerte deuten mit Ausprägungen, die jeweils kleiner als 1,5 sind, auf eine relativ gute Anpassung der erhobenen Daten an die jeweils ermittelten Verteilungen hin. Darüber hinaus zeigt Tabelle 6 die Parameter arithmetisches Mittel (mean), Median, Modus, Standardabweichung (standard deviation), Varianz (variance), Schiefe (skeweness) und Wölbung (kurtosis) für jede der geschätzten Verteilungen. 


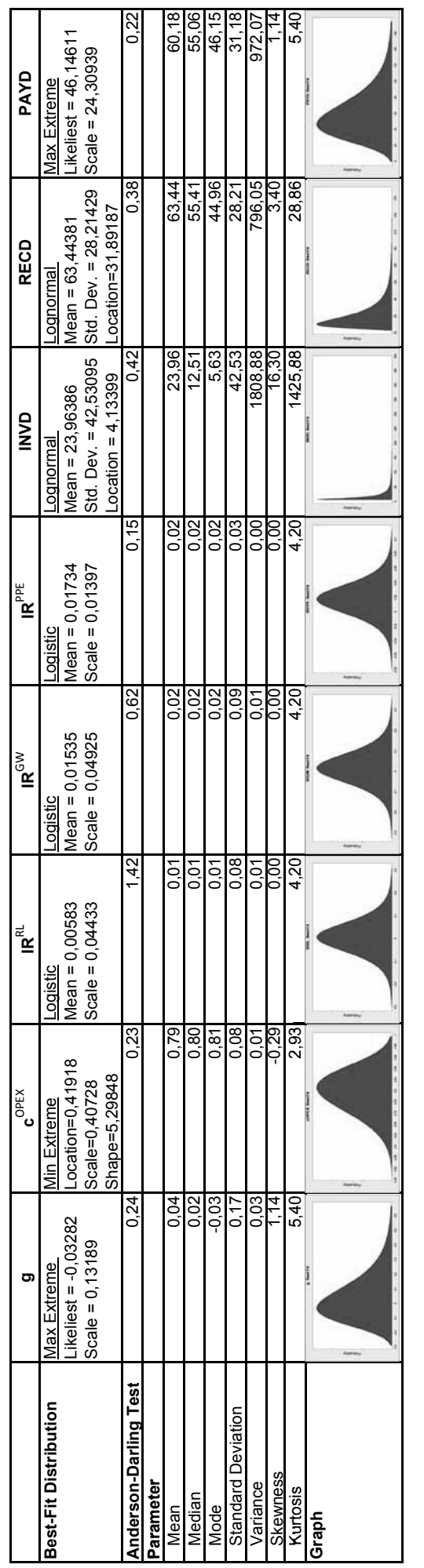

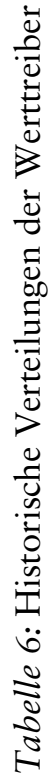




\subsection{Abbildung von Korrelationen}

Bevor die ermittelten Verteilungen zur Simulation der bewertungsrelevanten Free Cash Flows genutzt werden, gilt es, die Korrelationen der Werttreiber paarweise zu erfassen (Damodaran 2009, 20). Die Abbildung der Korrelationen zwischen den identifizierten Werttreibern bildet neben der Schätzung der Verteilungen die grösste konzeptionelle Schwierigkeit im Rahmen der Risikosimulation (Brealey et al. 2008, 280). Technisch erfolgt dies relativ problemlos, indem softwaregestützt eine Korrelationsmatrix für die identifizierten Werttreiber erstellt wird, die den Gleichlauf zwischen den Variablen darstellt. Aber die ermittelten Korrelationen gilt es zu plausibilisieren, bevor sie im Modell berücksichtigt werden (vgl. ausführlich Vose 1996, $191 \mathrm{ff}$.).

Allgemein können Korrelationen zwischen statistischen Variablen auf drei Gründe zurückgeführt werden (Vose 1996, 192). Erstens kann ein ökonomischer Zusammenhang zwischen den Variablen bestehen. Zweitens können externe, ausserhalb des Modells liegende, Grössen existieren, die die Variablen beeinflussen. Drittens kann zufällig eine Korrelation zwischen zwei Variablen bestehen. Tabelle 7 zeigt die ermittelten paarweisen Korrelationen der Werttreiber auf Basis des Jahres 2007, die im Fallbeispiel berücksichtigt werden.

Für diese lässt sich zum einen ein ökonomischer Zusammenhang identifizieren. Zum anderen kann für sie jeweils die Nullhypothese „Der Korrelationskoeffizient der identifizierten Werttreiber ist gleich Null“ mit Hilfe statistischer Testverfahren abgelehnt werden. Folglich wird von einer begründbaren und statistisch signifikanten Korrelation der angeführten Variablen für das Fallbeispiel ausgegangen. Die unterstellten ökonomischen $\mathrm{Zu}$ sammenhänge zwischen den Werttreibern, die durch deren Korrelationen abgebildet werden, sind nachfolgend beschrieben. Zeitliche Abhängigkeiten der Variablen werden vereinfachend nicht unterstellt.

- Höhere Wachstumsraten des Umsatzes $g_{t}$ gehen mit höheren Investitionsraten in den Firmenwert $I R_{t}^{G W}$ einher. Als Beispiel sind M\&A-Aktivitäten zu nennen, aus denen höheres Umsatzwachstum resultiert und bei entsprechenden Kaufpreisen ein höherer Firmenwert ausgewiesen wird.

- Hohe Wachstumsraten des Umsatzes $g_{t}$ gehen mit hohen Investitionsraten in Sachanlagen $I R_{t}^{P P E}$ einher. Als Beispiel seien Erweiterungsinvestitionen genannt, die zu höheren Umsatzwachstumsraten führen.

- Steigende operative Kostenquoten $c_{t}^{\text {OPEX }}$ gehen mit hohen Investitionsraten in Rechte und Lizenzen $I R_{t}^{R L}$ einher. Somit wird unterstellt, dass Wachstum zunächst mit sinkender Profitabilität, d. h. mit höheren Kostenquoten, einhergeht.

- Steigende operative Kostenquoten $c_{t}^{O P E X}$ gehen mit einer verlängerten Umschlagsdauer der Forderungen $R E C D_{t}$ einher. Somit wird unterstellt, dass höhere operative Kosten zu einem Forderungsaufbau und längeren Umschlagsdauern führen.

- Steigende Investitionsraten bei Rechten und Lizenzen $I R_{t}^{R L}$ gehen mit steigenden Investitionen in den Firmenwert $I R_{t}^{G W}$ einher. Beispiele sind M\&A-Aktivitäten und Investiti- 
onsprogramme, die zugleich Investitionen in Rechte, Lizenzen und Firmenwert beinhalten.

- Steigende Investitionsraten bei Rechten und Lizenzen $I R_{t}^{R L}$ gehen mit steigenden Investitionen in Sachanlagen $I R_{t}^{P P E}$ einher. Als Beispiel seien Investitionsprogramme erwähnt, die zugleich Investitionen in Rechte, Lizenzen und Sachanlagen beinhalten.

- Steigende Investitionsraten beim Firmenwert $I R_{t}^{G W}$ gehen mit steigenden Investitionsraten bei Sachanlagen $I R_{t}^{P P E}$ einher. Beispiele sind M\&A-Aktivitäten und Investitionsprogramme, die mit Investitionen in Sachanlagen und Firmenwerte einhergehen.

- Steigende Zahlungsziele für Vorräte $I N V D_{t}$ gehen mit steigenden Zahlungszielen für Verbindlichkeiten $P A Y D_{t}$ einher. Somit wird unterstellt, dass der geringere Vorratsumschlag durch spätere Zahlungen an Lieferanten finanziert wird.

\subsection{Durchführung der Monte-Carlo-Simulation}

Sind die Korrelationen der Werttreiber im Modell hinterlegt, können mit Hilfe einer Monte-Carlo-Simulation die historischen Verteilungsdaten in einem Analogieschluss auf das Bewertungsobjekt übertragen werden. Somit werden die Werttreiber für das Bewertungsobjekt mittels Zufallsziehung aus den geschätzten historischen Verteilungen generiert und als Inputgrössen mit dem Modell zur integrierten Finanzplanung verknüpft. Die Stichprobenziehung erfolgt mittels Crystal Ball. Dadurch werden „automatisch“ Verteilungen für die bewertungsrelevanten unverschuldete Free Cash Flows $F C F_{t}^{u}$ erzeugt. Das bedeutet, dass unter Rückgriff auf die Ergebnisse der Risikoanalyse Erwartungswerte für die unverschuldeten Free Cash Flows gebildet werden.

Dabei ist darauf zu achten, dass jedes simulierte Szenario grundsätzlich konsistent und beobachtbar sein muss. Zudem sollte die Anzahl der Durchläufe hinreichend gross gewählt werden. Dabei gilt (Damodaran 2009, 17): Je grösser die Anzahl der stochastischen Inputvariablen, je höher die Verschiedenheit der Verteilungen und je grösser die potentielle Bandbreite an Werten, desto grösser sollte die Anzahl der Simulationsdurchläufe sein. Nach 10.000 Simulationsdurchläufen ergeben sich für die zehn Jahre der Detailplanungsphase stabile periodische Free Cash Flow-Verteilungen.

Die simulierten Verteilungen machen das Risiko der zukünftigen Entwicklung der unverschuldeten Free Cash Flows transparent. Die Parameter der simulierten Verteilungen sind in Tabelle 8 zusammengefasst. Anhand der periodischen Verteilungen und ihrer Parameter kann die Erwartungsbildung und letztlich die periodische Verdichtung auf Erwartungswerte für die eigentliche Durchführung der Unternehmensbewertung erfolgen. 

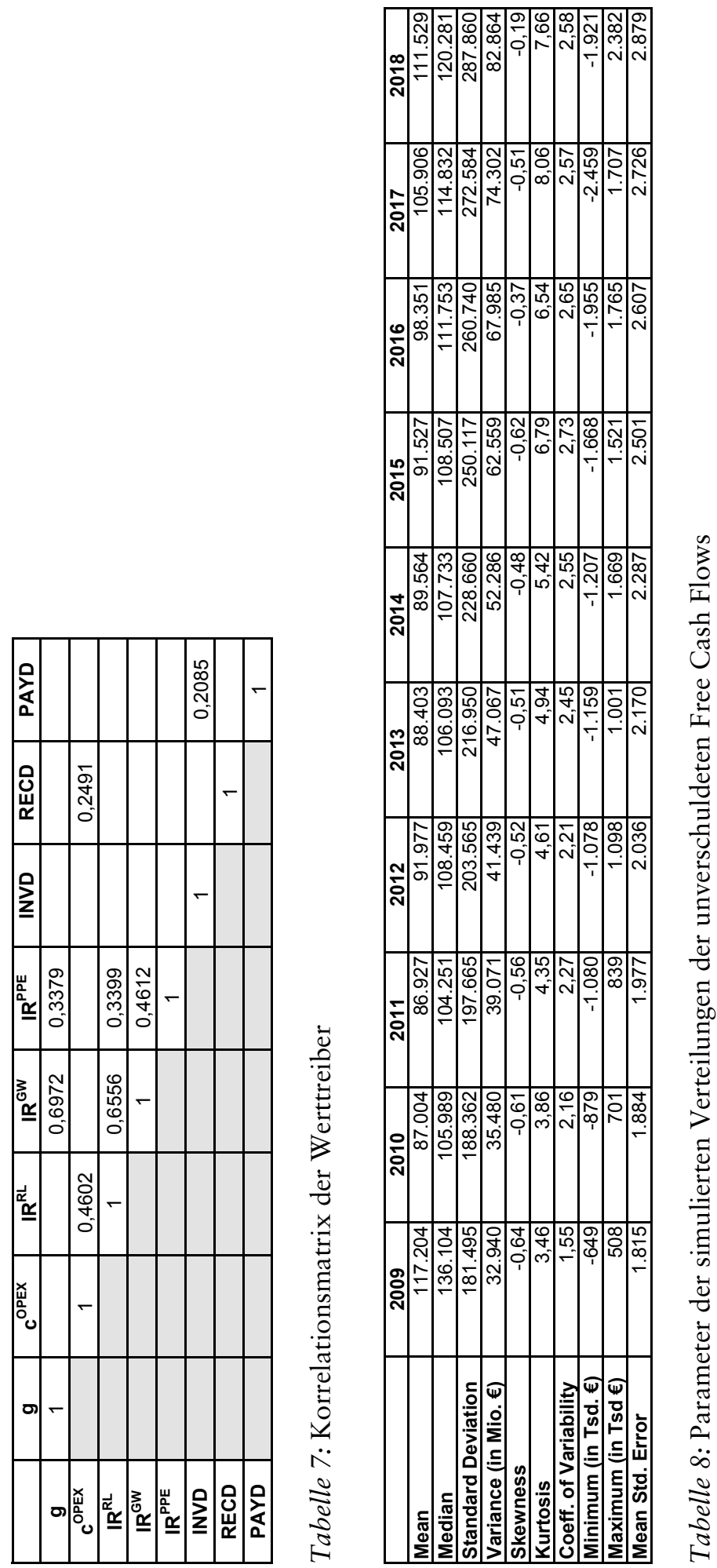


\section{Durchführung der Unternehmensbewertung}

\subsection{Vorgehensweise}

Erfolgt die Unternehmensbewertung in einem Zweiphasen-Modell, ${ }^{13}$ gilt es, die gebildeten Erwartungswerte der unverschuldeten Free Cash Flows für die Detailplanungsphase unter Berücksichtigung des Risikos zu diskontieren. Zudem sind plausible Annahmen für die Wertentwicklung nach dem Detailplanungshorizont in der Fortführungsphase (Terminal Value) zu treffen (vgl. statt vieler Steiner/Wallmeier 1999, 2). Um eine fundierte Bewertung unter Abbildung der Fortführungsphase zu ermöglichen, wird das in Abschnitt 3.2 vorgestellte Modell nach Koller et al. (2005) verwendet. Dadurch kann die geplante Wertschaffung in der Fortführungsphase adressiert werden. Folgende Bewertungsgleichung wird zugrunde gelegt:

$$
E\left(E V_{t}\right)=\sum_{t=1}^{T} \frac{E\left(F C F_{t}^{u}\right)}{\left(1+W A C C_{t}\right)^{t}}+\frac{E\left(\operatorname{NOPLAT}_{T}^{u}\right)\left(1+g_{N}\right)\left(1-\frac{g_{N}}{R O I C_{I}}\right)}{\left(1+W A C C_{T}\right)^{T}\left(W A C C_{T}-g_{N}\right)}
$$

Die Bewertungsgleichung verdeutlicht, dass es sich bei den bewertungsrelevanten Zählergrössen um Erwartungswerte handelt. Im Rahmen der Risikozuschlagsmethode werden sie mit einem risikoadjustierten Kapitalisierungszins diskontiert (Schultze 2003, 263). Für die in der Praxis am weitesten verbreitete DCF-Methode nach dem WACC-Ansatz ist dies ein durchschnittlich gewichteter Kapitalkostensatz (Drukarczyk/Schüler 2003, 347), der in der Regel komponentenweise bestimmt wird (Ballwieser 2002, 737). Der Risikozuschlag für die Eigenkapitalkosten kann hierbei ohne explizite Kenntnis der Risikonutzenfunktion der Entscheidungsträger aus Kapitalmarktdaten mittels CAPM bzw. bei einem nicht-börsennotierten Unternehmen durch einen Analogieschluss ermittelt werden (Schultze 2003, $263 \mathrm{ff}$.$) .$

Die Anwendung des WACC-Ansatzes setzt zudem idealisierte Prämissen einer „wertorientierten“ oder einer „autonomen“ Verschuldungspolitik für das Bewertungsobjekt voraus (Brealey et al. 2008, 538 ff.). Im Fallbeispiel wird für die konsistente Anwendung des WACC-Ansatzes von einer wertorientierten Verschuldungspolitik ausgegangen, d. h. es wird ein konstantes Verhältnis von Eigen- und Fremdkapital zu Marktwerten unterstellt. Diese Voraussetzung wird im Fallbeispiel vereinfachend durch die Annahme einer langfristigen Zielkapitalstruktur mit 60\% Eigenkapital und 40\% Fremdkapital zu Marktwerten im Zeitpunkt $t=2018$ approximiert.

Unter dieser idealisierten und vereinfacht im Modell umgesetzten Finanzierungsprämisse lassen sich steuerliche Effekte aus anteiliger Fremdfinanzierung innerhalb der Kapitalkosten WACC $_{t}$ erfassen (Steiner/Wallmeier 1999, 3). ${ }^{14}$ Zudem wird von einem konstanten operativen Risiko des Bewertungsobjekts ausgegangen. Im Fallbeispiel werden Eigenkapital-

13 Grundsätzlich sind bei der Phaseneinteilung strategische Zusammenhänge und der Grad an verfügbaren Informationen zu beachten, vgl. Schultze (2003, $240 \mathrm{ff}$.$) .$

14 Annahmegemäss wird davon ausgegangen, dass die Zinsschranke nicht greift. Siehe Förster et al. (2009, 985-1018) für ein Bewertungsmodell bei einem möglichen Eingreifen der Zinsschranke. 
kosten von $8,66 \%{ }^{15}$ und Fremdkapitalkosten nach Steuern von 5,02\% ${ }^{16}$ unterstellt. Daraus errechnet sich ein konstanter WACC in Höhe von 7,20\% $(8,66 \% * 60 \%+$ 5,02\%*40\%). Unter diesen Annahmen werden die simulierten und zu Erwartungswerten verdichteten Free Cash Flows aus Tabelle 8 bewertet.

Die Schätzung des Terminal Value erfolgt auf Basis des operativen Gewinns NOPLAT ${ }_{t}^{u}$ für das letzte Planjahr 2018, der erwarteten nachhaltigen nominalen Wachstumsrate $g_{N}$

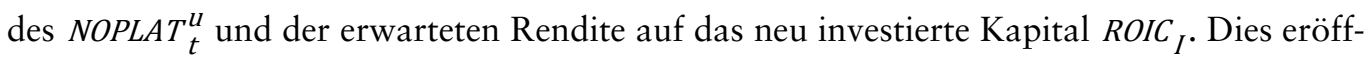
net die Möglichkeit, Annahmen zur Wertschaffung jenseits des Detailplanungshorizonts ab 2019 zu modellieren. Liegen die Kapitalrenditen des neu investierten Kapitals ROIC $_{I}$ oberhalb der Kapitalkosten $W A C C_{t}$, wird unterstellt, dass auch jenseits des Detailplanungshorizonts Investitionsprojekte mit positivem Kapitalwert durchgeführt werden können (Koller et al. 2005, 135 ff.). Im Fallbeispiel wird von einer nominalen Wachstumsrate des NOPLAT $_{t}^{u}$ in Höhe von $1,00 \%$ und einer Kapitalrendite ROIC $_{I}$ in Höhe von 8,00\% ausgegangen, die wenig oberhalb der unterstellten Kapitalkosten von 7,20\% liegt. Darin spiegeln sich die aktuellen langfristigen Marktwachstums- und Wettbewerbsprognosen für die Verlagsbranche wieder. Das bedeutet, dass zwar ein wertschaffendes Wachstum unterstellt wird. Der sogenannte competitive advantage wird indes als gering angenommen, weil der Spread der Kapitalrendite des zukünftig investierten Kapitals über den unterstellten Kapitalkosten nur gering ausfällt. Implizit wird also von einem begrenzten zukünftigen Potential ausgegangen.

Unter diesen Annahmen ergibt sich ein erwarteter Brutto-Unternehmenswert $E\left(E V_{t}\right)$ für das fiktive Verlagsunternehmen in Höhe von 1.897.014 Mio. € zum Bewertungsstichtag $t=2009$ entsprechend der Darstellung in Tabelle 9 .

\subsection{Mehrgewinn der dargestellten Vorgehensweise und Sensitivitätsbetrachtungen}

Der auf Basis durchschnittlicher historischer Werttreiber nach einer Monte-Carlo-Simulation ermittelte Brutto-Unternehmenswert weicht von dem Brutto-Unternehmenswert ab, der sich ergibt, wenn der Bewertung die Free Cash Flows zugrundelegt werden, die sich in der integrierten Finanzplanung auf Basis der durchschnittlichen historischen Werttreiber vor Durchführung einer Monte-Carlo-Simulation ergeben. In diesem Fall beträgt der Brutto-Unternehmenswert $E\left(E V_{t}\right)$ 2.196.689 Mio. € zum Bewertungsstichtag $t=2009$ (siehe Tabelle 10) und liegt ca. 15,8\% über dem Wert, der sich aus den verdichteten Free Cash Fows der simulierten Verteilungen ergibt.

15 Die Eigenkapitalkosten ergeben sich unter Verwendung einer konstanten Marktrisikoprämie von $5,00 \%$. Auf diesen Wert wird ein errechneter Betafaktor von 1,101 angewandt. Die 1,101 spiegeln einen Business Risk Index, also ein Asset Risiko bei reiner Eigenfinanzierung, von 0,738 wieder, der aus der Vergleichsgruppe börsennotierter Zeitungs- und Zeitschriftenverlage abgeleitet ist. Die risikolose Verzinsung wird mit 3,15\% angenommen. Es ergibt sich 5,00\%*1,101+3,15\%=8,66\%.

16 Die Fremdkapitalkosten ergeben sich aus der Annahme von Fremdfinanzierungskosten vor Steuern von $6,81 \%$. Bei einem Steuersatz von $26,325 \%$ ergibt sich ein Fremdkapitalkostensatz nach Steuern von $5,02 \%(6,81 \% *(1-0,26325)=5,02 \%)$. Dabei wird der im deutschen Steuersystem gültige Zusammenhang aus Gleichung (12) unterstellt sowie die Regelung, dass für die Bestimmung der Gewerbesteuerbemessungsgrundlage $25 \%$ der für die Körperschaftsteuerberechnung abgezogenen Fremdkapitalzinsen dem Gewerbeertrag hinzuzurechnen sind. Es ergibt sich $15 \% *(1+5,5 \%)+3,5 \% * 400 \%$ $* 0,75=26,325 \%$. 


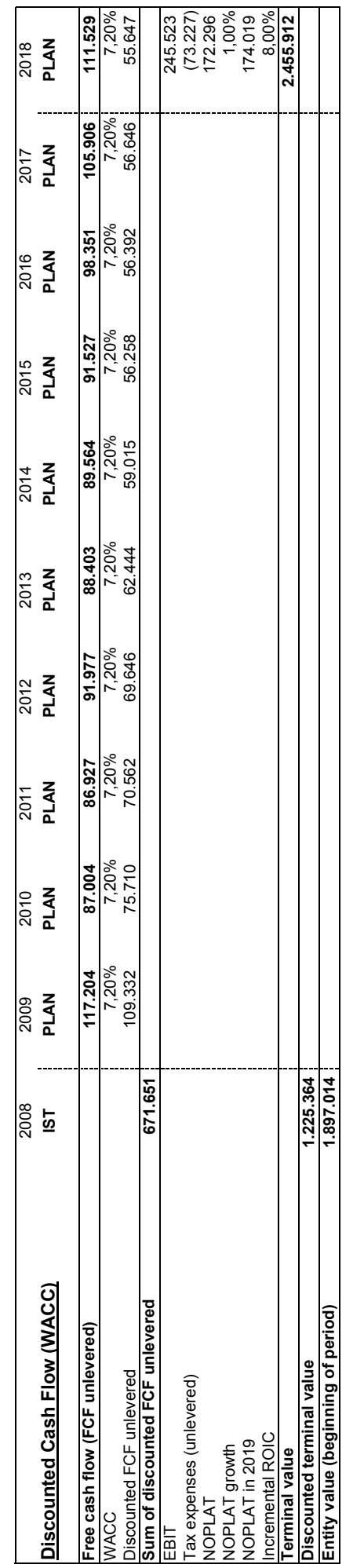

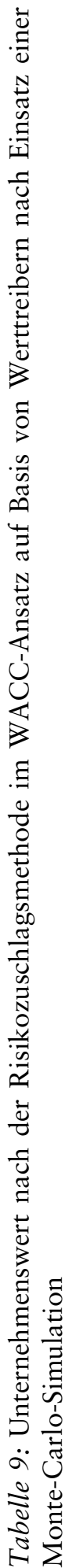

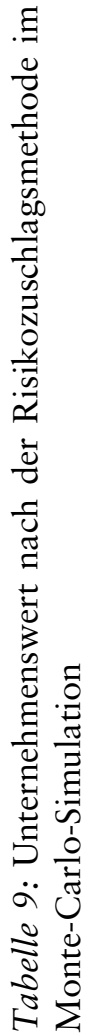

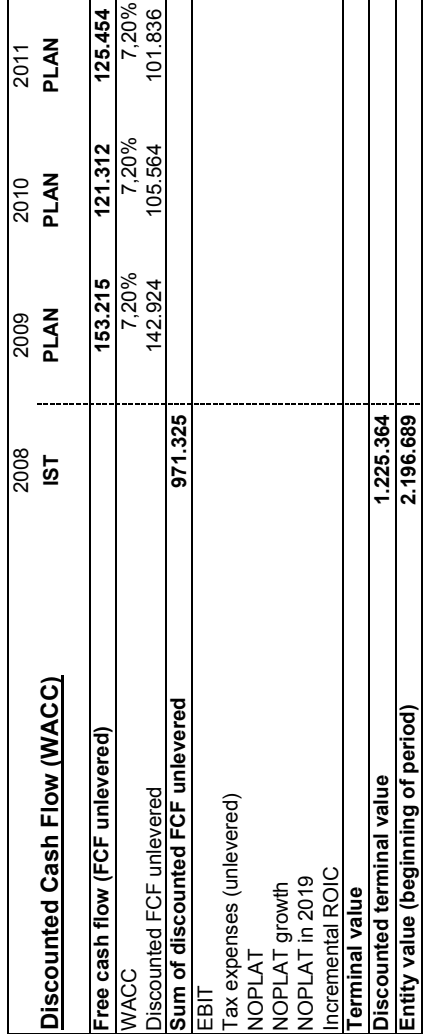

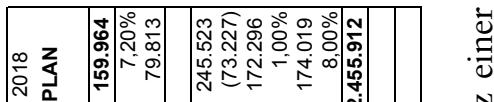

$z=\frac{1}{0}$

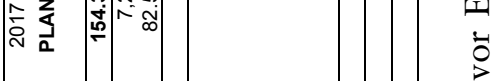

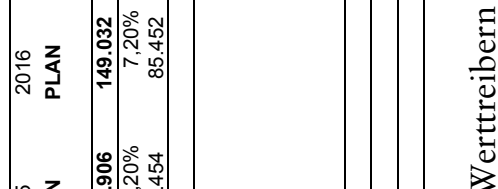

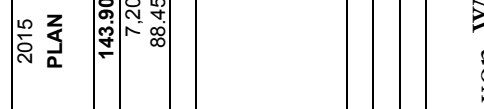

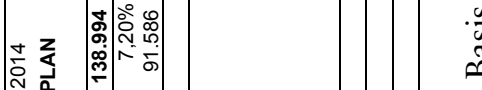

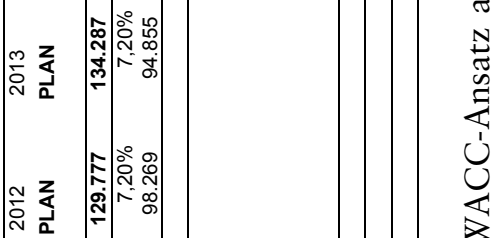

$\Xi$

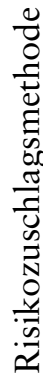

ț

兘

这

5

छี

离

जि 
Hierin zeigt sich der Mehrgewinn der dargestellten Vorgehensweise. Verwendet man durchschnittliche historische Wertreiber als Basis, kann es zu einer anderen Berechnung des Unternehmenswerts kommen, d. h. sowohl zu einem höheren als auch niedrigen Betrag. Der Beispielfall zeigt aufgrund der Verteilungsannahmen der Werttreiber einen höheren Wert. Durch die Monte-Carlo-Simulation der Werttreiber ergeben sich tiefergehende Einblicke in das Risikoprofil der bewertungsrelevanten finanziellen Überschüsse. Somit verbindet die Vorgehensweise die Vorteile der Fundamentalbewertung mit den Erkenntnissen einer Risikobetrachtung, weil die spezifisch für das Bewertungsobjekt aus Verteilungsschätzungen abgeleiteten Free Cash Flows Grundlage der Bewertung sind. Dem Grundgedanken der Multiplikatorverfahren folgend werden die für die Monte-Carlo-Simulation benötigten Verteilungsschätzungen aus den historischen Daten von Vergleichsunternehmen gewonnen. Damit wird nicht naiv eine Normalverteilung der zu simulierenden Grössen unterstellt, sondern realitätsnäher und daher mit grösserer praktischer Relevanz agiert.

Beachtung gilt es der Tatsache zu schenken, dass die Wahl des jeweiligen Stichprobenjahrs zur Bildung von Verteilungshypothesen für die Werttreiber einen massgeblichen Einfluss auf das Bewertungsergebnis hat. Dies sei exemplarisch mit folgender Sensitivitätsbetrachtung illustriert. Hält man am Bewertungsstichtag $t=2009$ fest, variiert aber das Stichprobenjahr von 2005 bis 2008, ergeben sich die Brutto-Unternehmenswerte in Tabelle 11. Zwar sind die ermittelten Verteilungsannahmen robust, aber die maximale Differenz der Unternehmenswerte im Verhältnis zum hier im Detail hergeleiteten Unternehmenswert basierend auf dem Stichprobenjahr 2007 beträgt ca. 32,4\%.

\begin{tabular}{|l|r|}
\hline Stichprobenjahr & Brutto-Unternehmenswert $€$ \\
\hline 2005 & 1.520 .710 \\
\hline 2006 & 1.386 .239 \\
\hline 2007 & 1.897 .014 \\
\hline 2008 & 1.281 .675 \\
\hline
\end{tabular}

Tabelle 11: Sensitivitätsbetrachtung der Brutto-Unternehmenswerte bei Variation der Stichprobenjahre zur Bildung von Verteilungshypothesen für die Werttreiber

Im Zusammenhang mit der Ableitung einer ausreichend grossen Anzahl von Unternehmenswerten ergäbe sich dann grundsätzlich auch die Möglichkeit, die statistische Signifikanz der Unterschiede zu prüfen. Sicher wird der Praktiker seine Analyse nicht bei der Punktschätzung des Unternehmenswerts stoppen, auch wenn diese Punktschätzung das Risikoprofil der bewertungsrelevanten finanziellen Überschüsse abzubilden versucht. Der Bewerter muss sich über Wertspannen bewusst sein, auch bei Verwendung von stochastischen Risikoanalysen.

Insbesondere für die praktische Anwendung ergeben sich aus der Sensitivitätsbetrachtung wichtige Überlegungen. Da eine Risikoanalyse immer dann einen Mehrwert bringen kann, wenn eine Bewertungssituation durch besonders hohe Unsicherheit - wie im Fallbeispiel der strukturellen Wandel der Verlagsbranche - geprägt wird, ist bei der Ableitung aus Vergangenheitsdaten besondere Sorgfalt angebracht. Dabei ist die Frage zu beantworten, wie repräsentativ Vergangenheitsbeobachtungen für die Zukunft sind. Dies gilt im Besonderen auch für das in der Praxis stark an Bedeutung gewinnende Korrelationsrisiko. So 
wird im konkreten Anwendungsfall anders als in diesem Beitrag nicht ein Vergangenheitsjahr exemplarisch gewählt werden können. Vielmehr sind zukunftsgerichtete Anpassungen an den historischen Daten des Bewertungsobjekts und der gewählten Vergleichsunternehmen vorzunehmen. Dies ist Voraussetzung, um eine repräsentative Ausgangsbasis für deren Extrapolation zu schaffen. Das exemplarisch gewählte Beispiel führt das Spannungsfeld für Bewerter und Entscheidungsträger besonders deutlich vor Augen. Einerseits befindet sich die Branche aufgrund der Markt- und Wettbewerbssituation in einem strukturellen Wandel, was berechtigte Kritik an der Fortschreibung von (angepassten) historischen Verteilungen hervorruft. Andererseits vermitteln die (angepassten) historischen Verteilungen und Korrelationen tiefergehende Einblicke in das Risikoprofil der bewertungsrelevanten finanziellen Überschüsse.

Im Kontext von zukunftsgerichteten Anpassungen muss sich der Bewerter darüber hinaus im Klaren sein, dass es auch known unknowns gibt. Das bedeutet, dass der Bewerter zwar darum weiss, dass Umstände vorliegen, die letztlich das Bewertungsergebnis beeinflussen, er diese aber nicht kennt. Ebenso muss sich der Bewerter um die Existenz der von Taleb (2007) Black Swans genannten Ereignisse bewusst sein. ${ }^{17}$ Solche Ereignisse treten ungerichtet und unvorhersehbar auf und können ausserordentlich starke Auswirkungen nach oben und unten zeigen. Taleb selbst nennt das Internet einen Black Swan, dessen zunehmende Verbreitung auch den strukturellen Wandel der Printmedienbranche massiv beeinflusst.

Schliesslich bietet es sich in der praktischen Anwendung der vorgestellten Vorgehensweise an, das Model einer Validierung zuzuführen. Das heisst, dass die simulierten Bewertungsergebnisse einen Abgleich mit realisierten Werten erfahren. Hierzu ist es denkbar, dass einerseits Marktwerte genommen werden. Dies bedeutet, dass man die Marktkapitalisierung zuzüglich der Nettoschuldenposition ermittelt und mit dem Bewertungsergebnis des Modells vergleicht. Im unterstellten Beispielfall wird indes von einem nicht-notierten Unternehmen ausgegangen. Hier wären dann andererseits und soweit verfügbar Transaktionswerte zur Validierung zu verwenden. Ziel dieser Vorgehensweise ist die Einordnung der Modellergebnisse und gegebenenfalls die Ermittlung von Anhaltspunkten für zukunftsgerichtete Anpassungen an den historischen Daten.

\section{Zusammenfassung}

Im vorliegenden Beitrag wird gezeigt, wie stochastische Risikoanalysen für die Unternehmensbewertung durchgeführt werden können. Es wird schrittweise dargestellt, wie unter Rückgriff auf historische Daten Verteilungsannahmen für relevante Werttreiber gebildet werden können. Anders als in einigen deutschen Beiträgen (z. B. Coenenberg 1970; von Weizsäcker/Krempel 2004; Jödicke 2007, 166-171) zur Nutzung der Monte-Carlo-Simulation in der Unternehmensbewertung wird sie nicht zur Verteilungsbildung für den Unternehmenswert genutzt. Vielmehr wird sie zur Schätzung von Verteilungen der den bewertungsrelevanten finanziellen Überschüssen - definiert als unverschuldete Free Cash Flows - vorgelagerten Werttreibern eingesetzt. Theoretisch könnten zwar auch die unverschulde-

17 Vollständigkeitshalber sei darauf verwiesen, dass Taleb (2007) neben den Eigenschaften Aussreisser und extreme Auswirkungen auch die Eigenschaft, dass solche Ereignisse nach dem Auftreten, also ex post vorhersagbar im Sinne von erklärbar werden, zur Charakterisierung verwendet. 
ten Free Cash Flows direkt simuliert werden. In der Praxis fehlt es aber am Bezugspunkt für die Ableitung von Verteilungen und Korrelationen derselben.

Die stochastische Risikosimulation wird somit Instrument zur Schätzung der Zählergrössen in der Bewertungsgleichung (vgl. nachfolgend Brealey et al. 2008, 281-282). Die Bewertung selbst wird nach der in der Praxis gängigen Risikozuschlagsmethode vorgenommen. Wird der Risikozuschlag wie in diesem Beitrag auf Basis des CAPM ermittelt, wird implizit unterstellt, dass nur für die Übernahme systematischer Risiken eine Risikoprämie erwartet werden darf, während unsystematische Risiken durch Diversifikation zu eliminieren sind.

Das exemplarisch dargestellte Verfahren zur Durchführung einer stochastischen Unternehmensbewertung mittels Monte-Carlo-Simulation bedarf im konkreten Anwendungsfall - je nach Bewertungsanlass und -zweck - spezifischer Anpassungen und methodischer Verfeinerungen. Insbesondere die bekannte Tatsache, dass historische Verteilungen und Korrelationen nicht naiv in die Zukunft extrapoliert werden sollten, verdeutlicht dies.

Zugleich zeigt sich, dass das dargestellte Vorgehen die Unternehmensbewertung in Hinblick auf die Risikoerfassung erweitern kann. Es gilt aber auch, dass zahlreich aufgezeigte Aspekte in weiteren Forschungsüberlegungen vertieft werden müssen. So sind Verfeinerungen der statistischen Verfahren und Methoden zur Schätzung der Verteilungen und Korrelationen im Allgemeinen und im jeweiligen branchenspezifischen Anwendungskontext zu prüfen. Zudem können neben der Verwendung von Querschnittsdaten auch Zeitreihendaten für die stochastischen Simulationen genutzt werden. Eine vertiefende Analyse könnte dann auch zeigen, ob die hier beobachtete Berechnung eines höheren Unternehmenswerts auf Basis von Plangrössen vor einer Simulation regelmässig auftritt oder ob sich auch niedrigere Werte zeigen. Je nach Datenverfügbarkeit kann zudem eine detailliertere Abbildung und Simulation der Werttreiber erfolgen, z. B. durch Erfassung von geschäftsmodellspezifischen Preis- / Mengengerüsten. Für das integrierte Finanzmodell können zudem kennzahlenbasierte Abbruchs- und Nichtnegativitätsbedingungen sowie bestimmte Schwellenwerte festgelegt werden, die im Rahmen der Simulationen zu berücksichtigen sind, z. B. um Covenants abzubilden. Darüber hinaus ist auch für die Schätzung der kritischen Parameter Wachstum und Rentabilität für den Fortführungswert grundsätzlich die Nutzung von empirischen Daten und stochastischen Simulationen denkbar.

\section{Literaturhinweise}

Ballwieser, W. (1990): Unternehmensbewertung und Komplexitätsreduktion, 3. Aufl., Wiebaden.

Ballwieser, W. (2007): Unternehmensbewertung - Prozess, Methoden und Probleme, 2. Aufl., Stuttgart.

Bamberg, G./Baur, F./Krapp, M. (2007): Statistik, 13. Aufl., München.

Bamberg, G./Dorfleitner, G./Krapp, M. (2004): Zur Bewertung risikobehafteter Zahlungsströme mit intertemporaler Abhängigkeitsstruktur, in: Betriebswirtschaftliche Forschung und Praxis, Jg. 56, Nr. 2, S. 101-118.

Bamberg, G./Dorfleitner, G./Krapp, M. (2006): Unternehmensbewertung unter Unsicherheit - Zur entscheidungsorientierten Fundierung der Risikoanalyse, in: Zeitschrift für Betriebswirtschaft, Jg. 76, Nr. 3, S. 287-307. 
Buch, A./Dorfleitner, G. (2007): Ein Vergleich der Sicherheitsäquivalentmethode und der Risikoanalyse als Methoden zur Bewertung risikobehafteter Zahlungsströme, in: Zeitschrift für Betriebswirtschaft, Jg. 77, Nr. 2, S. 141-170.

Brealey, R.A./Myers, S.C./Allen, F. (2008): Principles of Corporate Finance, 9. Aufl., New York et al.

Coenenberg, A.G. (1970): Unternehmensbewertung mit Hilfe der Monte-Carlo-Simulation, in: Zeitschrift für Betriebswirtschaft, Jg. 40, Nr. 12, S. 793-804.

Coenenberg, A.G. (1992): Unternehmensakquisition und Unternehmensbewertung, in: Busse von Colbe, W./Coenenberg, A.G. (Hrsg.): Unternehmensakquisition und Unternehmensbewertung, Stuttgart, S. 113-156.

Coenenberg, A.G./Schultze, W. (2002): Das Multiplikator-Verfahren in der Unternehmensbewertung: Konzeption und Kritik, in: Finanzbetrieb, Jg. 4, Nr. 12, S. 697-703.

Damodaran, A. (2009): The Dark Side of Valuation, Manuskript zur 2. Aufl., S. 18-19, http:// pages.stern.nyu.edu/ adamodar/pdfiles/DSV2/Ch3.pdf, Stand 6.7.2009.

Dorfleitner, G./Bamberg, G./Krapp, M. (2006): Unternehmensbewertung unter Unsicherheit - Zur entscheidungsorientierten Fundierung der Risikoanalyse, in: Zeitschrift für Betriebswirtschaft, Jg. 76, Nr. 3, S. 287-307.

Drukarczyk, J./Schüler, A. (2003): Kapitalkosten deutscher Aktiengesellschaften - eine empirische Untersuchung, in: Finanzbetrieb, Jg. 5, Nr. 6, S. 337-347.

Drukarczyk, J./Ernst, D. (2007): Branchenorientierte Unternehmensbewertung, 2. Aufl., München.

Förster, H.H./Rhode, C. (2009): Finanziellen Spielraum aktiv nutzen - Zeitungs- und Zeitschriftenverlage in konvergierenden Medienmärkten, Deloitte Research, Düsseldorf.

Förster, H.H./Stöckel, S./Brenken, H. (2009): Die Bedeutung der Zinsschranke für die Bewertung von Tax Shields in einem modifizierten APV-Ansatz unter Verwendung einer entsprechend angepassten Eigenkapitalkosten-Reaktionshypothese, in: Zeitschrift für Betriebswirtschaft, Jg. 79, Nr. 9, S. 985-1018.

Grob, H.L./Mrzyk, A. (1998): Risiko-Chancen-Analyse in der Investitionsrechnung - Integration von VOFI und Crystal Ball, in: Controlling, Jg. 10, S. 120-129.

IDW (2008) (Hrsg.): WP-Handbuch, Bd. I1, 13. Aufl., Düsseldorf.

Jödicke, D. (2007): Unternehmensbewertung mit vollständigen Finanzplänen - Vergleich zur Discounted Cashflow-Methode, Saarbrücken.

Keiber, K./Kronimus, A./Rudolf, M. (2002): Die Bewertung von Wachstumsunternehmen, in Zeitschrift für Betriebswirtschaft, Jg. 72, Nr. 7, S. 735-764.

Koller, T./Goedhart, M./Wessels, D. (2005): Valuation, 4. Aufl., New York et al.

Kruschwitz, L./Löffler, A. (2008): Kapitalkosten aus theoretischer und praktischer Perspektive, in: Die Wirtschaftsprüfung, Jg. 61, Nr. 17, S. 803-810.

Madrian, J./Auerbach, J. (2009): Zum Risikokalkül in der Unternehmensbewertung, in: Littkemann, J./Zündorf, H. (Hrsg.): Beteiligungscontrolling Band II, 2. Aufl., Herne/Berlin, S. 381-414.

Modigliani, F./Miller M.H. (1958): The Cost of Capital, Corporation Finance and the Theory of Investment, in: American Economic Review, Vol. 48, S. 261-297.

Perridon, L./Steiner, M. (2006): Finanzwirtschaft der Unternehmung, 14. Aufl., München.

Rappaport, A. (1998): Shareholder Value, 2. Aufl., New York.

Richter, F. (2005): Mergers \& Acquisitions, München. 
Rubinstein, R.Y./Kroese, D.P. (2008): Simulation and the Monte Carlo Method, 2. Aufl., New York et al.

Steiner, M./Wallmeier, M. (1999): Unternehmensbewertung mit Discounted Cash Flow-Methoden und dem Economic Value Added-Konzept, in: Finanz Betrieb, Jg. 1, Nr. 5, S. 1-10.

Schultze, W. (2003): Methoden der Unternehmensbewertung, 2. Aufl., Düsseldorf.

Schwartz, E.S./Moon, M. (2000): Rational Pricing of Internet Companies, in: Financial Analysts Journal, Vol. 56, Nr. 3, S. 62-75.

Taleb, N. (2007): The Black Swan, London.

von Weizsäcker, R.K./Krempel, K. (2004): Risikoadäquate Bewertung nicht-börsennotierter Unternehmen, in: Finanz Betrieb, Jg. 6, Nr. 12, S. 808-814.

Vose, D. (1996): Quantitative Risk Analysis: A Guide to Monte Carlo Simulation Modelling, Chichester.

Heinrich H. Förster, CFA, Dr., ist Lehrbeauftragter an der Fakultät für Wirtschaftswissenschaften der RWTH Aachen.

Anschrift: Lehr- und Forschungsgebiet Betriebswirtschaftslehre, insb. Entscheidungsforschung \& Finanzdienstleistungen, Templergraben 64, 52056 Aachen, Deutschland, Tel.: +49 (0)241/80-96174, Fax: +49 (0)241/80-92347, E-Mail: heinrich.foerster@live.com

Der Autor dankt Herrn Christian M. Rhode, CFA für seine Unterstützung sowie zwei anonymen Gutachtern für wertvolle Hinweise und Verbesserungsvorschläge. 